\title{
OSSOS. XII. Variability Studies of 65 Trans-Neptunian Objects Using the Hyper Suprime-Cam*
}

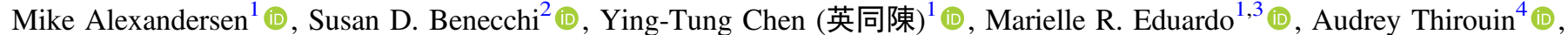 \\ Megan E. Schwamb ${ }^{5}$ (1) , Matthew J. Lehner ${ }^{1,6,7}$ (1) , Shiang-Yu Wang (王祥宇) ${ }^{1}$ (1), Michele T. Bannister ${ }^{8}$ (i), Brett J. Gladman ${ }^{9}$, \\ Stephen D. J. Gwyn ${ }^{10}$, JJ. Kavelaars ${ }^{10,11}$ (iD, Jean-Marc Petit ${ }^{12}$ (iD, and Kathryn Volk ${ }^{13}$ (i) \\ ${ }^{1}$ Institute of Astronomy and Astrophysics, Academia Sinica; 11F of AS/NTU Astronomy-Mathematics Building, No. 1 Roosevelt Rd., Sec. 4, Taipei 10617, Taiwan \\ mike.alexandersen@alumni.ubc.ca \\ ${ }^{2}$ Planetary Science Institute, 1700 E. Fort Lowell, Suite 106, Tucson, AZ 85719, USA \\ ${ }^{3}$ Department of Physical Sciences, University of the Philippines Baguio, Gov. Pack Rd., Baguio City, Benguet, 2600, Philippines \\ ${ }^{4}$ Lowell Observatory, 1400 W. Mars Hill Rd., Flagstaff, AZ 86001, USA \\ ${ }^{5}$ Gemini Observatory, Northern Operations Center, 670 North A'ohoku Pl., Hilo, HI 96720, USA \\ ${ }^{6}$ Department of Physics and Astronomy, University of Pennsylvania, 209 S. 33rd St., Philadelphia, PA 19104, USA \\ ${ }^{7}$ Harvard-Smithsonian Center for Astrophysics, 60 Garden St., Cambridge, MA 02138, USA \\ ${ }^{8}$ Astrophysics Research Centre, School of Mathematics and Physics, Queen's University Belfast, Belfast BT7 1NN, UK \\ 9 Department of Physics and Astronomy, University of British Columbia, Vancouver, BC V6T 1Z1, Canada \\ ${ }^{10}$ Herzberg Astronomy and Astrophysics Research Centre, National Research Council of Canada, 5071 W. Saanich Rd., Victoria, British Columbia V9E 2E7, Canada \\ ${ }^{11}$ Department of Physics and Astronomy, University of Victoria, Elliott Building, 3800 Finnerty Rd., Victoria, BC V8P 5C2, Canada \\ ${ }_{13}^{12}$ Institut UTINAM UMR6213, CNRS, Univ. Bourgogne Franche-Comté, OSU Theta F-25000 Besançon, France \\ ${ }^{13}$ Lunar and Planetary Laboratory, University of Arizona, 1629 E. University Blvd., Tucson, AZ 85721, USA \\ Received 2018 July 2; revised 2018 December 11; accepted 2019 March 7; published 2019 September 19
}

\begin{abstract}
We present variability measurements and partial light curves of trans-Neptunian objects (TNOs) from a two-night pilot study using Hyper Suprime-Cam (HSC) on the Subaru Telescope (Maunakea, Hawaii, USA). Subaru's large aperture $(8 \mathrm{~m})$ and HSC's large field of view $\left(1.77 \mathrm{deg}^{2}\right)$ allow us to obtain measurements of multiple objects with a range of magnitudes in each telescope pointing. We observed 65 objects with $m_{r}=22.6-25.5$ mag in just six pointings, allowing 20-24 visits of each pointing over the two nights. Our sample, all discovered in the recent Outer Solar System Origins Survey (OSSOS), spans absolute magnitudes of $H_{r}=6.2-10.8$ mag and thus investigates smaller objects than previous light curve projects have typically studied. Our data supports the existence of a correlation between the light curve amplitude and absolute magnitude seen in other works but does not support a correlation between the amplitude and orbital inclination. Our sample includes a number of objects from different dynamical populations within the trans-Neptunian region, but we do not find any relationship between variability and the dynamical class. We were only able to estimate periods for 12 objects in the sample and found that a longer baseline of observations is required for a reliable period analysis. We find that 31 objects (just under half of our sample) have variability of $\Delta_{\text {mag }}$ greater than 0.4 mag during all of the observations; in smaller $1.25 \mathrm{hr}, 1.85 \mathrm{hr}$, and $2.45 \mathrm{hr}$ windows, the median $\Delta_{\text {mag }}$ is $0.13,0.16$, and $0.19 \mathrm{mag}$, respectively. The fact that variability on this scale is common for small TNOs has important implications for discovery surveys (such as OSSOS or the Large Synoptic Survey Telescope) and color measurements.
\end{abstract}

Key words: Kuiper belt: general - minor planets, asteroids: general - planets and satellites: surfaces

Supporting material: extended figure, machine-readable tables

\section{Introduction}

Over the years, rotational light curves (the brightness variation versus time) from small bodies in our solar system have been used to derive physical properties about the shape, surface, cohesion, internal structure, and density of objects and to infer binarity (Sheppard et al. 2008; Thirouin et al. 2010; Benecchi \& Sheppard 2013). Small light-curve amplitudes can be caused by albedo variation across the surface of a spherical object, but moderate/large amplitude light curves (maximum minus minimum, $\Delta_{\mathrm{mag}} \geqslant 0.15-0.2 \mathrm{mag}$ ) are likely due to the shape of the object (Sheppard et al. 2008; Thirouin et al. 2010). Flat light curves can be explained by a nearly spherical object with a homogeneous surface or an elongated object with a poleon orientation. A flat light curve can also be due to an extremely slow rotational period undetectable during the

\footnotetext{
* Based on data collected at Subaru Telescope, which is operated by the National Astronomical Observatory of Japan.
}

observing window, and such a slow rotation may infer the presence of a companion (Thirouin et al. 2010, 2014; Benecchi \& Sheppard 2013). Alternatively, very large amplitude light curves $\left(\Delta_{\mathrm{mag}} \geqslant 0.9 \mathrm{mag}\right)$ with a U/V shape at the maximum/ minimum of the brightness are due to close/contact binary systems (Sheppard \& Jewitt 2004; Lacerda et al. 2014; Ryan et al. 2017; Thirouin et al. 2017; Thirouin \& Sheppard 2017, 2018).

In the trans-Neptunian belt, four main dynamical populations — classical, resonant, detached, and scattering objects-have been identified with additional subdivisions inside some of these broad groupings. Resonant objects are trapped in meanmotion resonance with Neptune, such that the orbital period (or mean-motion) of the trans-Neptunian object (TNO) and Neptune have a small integer ratio, which causes the TNO to experience stabilizing gravitational perturbations. Objects that are currently interacting strongly with Neptune in such a way that their orbits are unstable are called scattering (a semimajor 
Table 1

Field Centers Used in This Work

\begin{tabular}{|c|c|c|c|c|}
\hline $\begin{array}{l}\text { Field } \\
\text { Identifier }\end{array}$ & $\begin{array}{l}\text { R.A. } \\
\text { (hr) }\end{array}$ & $\begin{array}{l}\text { Decl. } \\
(\mathrm{deg})\end{array}$ & $\begin{array}{c}\text { Observations Night } 1 \\
\text { Useful/Total }\end{array}$ & $\begin{array}{c}\text { Observations Night } 2 \\
\text { Useful/Total }\end{array}$ \\
\hline LF07 & $00^{\mathrm{h}} 31^{\mathrm{m}} 33^{\mathrm{s}}$ & $+04^{\circ} 54^{\prime} 54^{\prime \prime}$ & $12 / 12$ & $10 / 12$ \\
\hline LF08 & $00^{\mathrm{h}} 41^{\mathrm{m}} 41^{\mathrm{s}}$ & $+05^{\circ} 17^{\prime} 52^{\prime \prime}$ & $12 / 12$ & $10 / 12$ \\
\hline LF09 & $00^{\mathrm{h}} 42^{\mathrm{m}} 34^{\mathrm{s}}$ & $+06^{\circ} 37^{\prime} 44^{\prime \prime}$ & $12 / 12$ & $10 / 12$ \\
\hline LF10 & $00^{\mathrm{h}} 45^{\mathrm{m}} 37^{\mathrm{s}}$ & $+07^{\circ} 59^{\prime} 02^{\prime \prime}$ & $12 / 12$ & $10 / 12$ \\
\hline LF11 & $01^{\mathrm{h}} 10^{\mathrm{m}} 00^{\mathrm{s}}$ & $+05^{\circ} 43^{\prime} 25^{\prime \prime}$ & $10 / 10$ & $9 / 10$ \\
\hline LF12 & $01^{\mathrm{h}} 21^{\mathrm{m}} 51^{\mathrm{s}}$ & $+06^{\circ} 34^{\prime} 20^{\prime \prime}$ & $10 / 10$ & $9 / 10$ \\
\hline
\end{tabular}

Note. On the second night, each field lost one or two observations due to low transparency.

axis change of at least $1.5 \mathrm{au}$ in $10 \mathrm{Myr}$ is required for this classification in the nomenclature of Gladman et al. 2008). The detached objects are those objects with perihelia that are so distant that they are beyond significant gravitational influence from Neptune; how they got there is a subject of active research. The resonant, detached, and scattering objects are all dynamically excited ("hot") populations, with wide inclination and eccentricity distributions, and are thought to have been caused by significant interaction with the giant planets or other small bodies during the era of planetary migration (Kaula \& Newman 1992; Malhotra 1993, 1995; Thommes et al. 2002; Kortenkamp et al. 2004; Hahn \& Malhotra 2005; Murray-Clay \& Chiang 2005; Tsiganis et al. 2005; Levison et al. 2008). The classical objects consists of nonresonant, non-scattering objects, primarily located at semimajor axes between 42 and $48 \mathrm{au}$. The classical population has both a hot subpopulation and a dynamically cold subpopulation of objects that have nearly circular orbits with low inclinations and eccentricities; the dynamically cold objects likely had only limited interactions with the giant planets. Due to their different formation mechanisms, it is possible that these dynamical populations have different light-curve properties, just as they have different color distributions (Trujillo \& Brown 2002; Peixinho et al. 2003; Fraser \& Brown 2012; Sheppard 2012; Tegler et al. 2016; Pike et al. 2017).

Because light curves of TNOs have been studied by several surveys over the past decades, it is now possible to study the rotational properties of the different dynamical populations. Several studies have identified different correlations/anticorrelations between orbital and physical/rotational properties, allowing us to understand the different dynamical environments in the trans-Neptunian belt (Duffard et al. 2009; Benecchi \& Sheppard 2013; Thirouin et al. 2016). However, most of the past surveys focused on bright objects, as they are the easiest ones to study with small/moderate aperture facilities. Therefore, we present a pilot study using the Subaru/Hyper Suprime-Cam (HSC) to study the rotational properties of the faint TNOs $\left(H_{r}>8.0\right.$ mag). We aim to explore the rotational properties of TNOs in several size ranges, particularly for smaller objects around the break in each population's size distribution (Shankman et al. 2013; Fraser et al. 2014; Alexandersen et al. 2016; Lawler et al. 2018); however, firm conclusions will require additional observations.

\section{Sample and Observations}

Our sample consists entirely of objects discovered or detected in the Outer Solar System Origins Survey (OSSOS; Bannister et al. 2016, 2018). OSSOS was a large program on the Canada-France-Hawaii Telescope (CFHT), which ran from 2013 through 2017. OSSOS detected and tracked more than 800 trans-Neptunian objects with regular follow-up observations during at least two years to determine accurate orbits and secure dynamical classifications (Bannister et al. 2018). Due to the design and success of OSSOS, we have very complete information on the orbital characteristics and classification of these objects. Likewise, because these objects were discovered recently, they are still tightly clustered on the sky, having only slightly diffused apart due to Kepler shear. This allows us to observe up to 17 objects at once within the $1.77 \mathrm{deg}^{2}$ field of view of Subaru Telescope's HSC (Miyazaki et al. 2018). The six fields used in this study (coordinates in Table 1) were chosen in order to maximize the number of objects within each field (see Figure 1), for a total of 63 TNOs (37 classical, 15 resonant, 5 detached, and 6 scattering) and two Centaurs spanning apparent magnitudes of $m_{r}=22.6-25.5 \mathrm{mag}$ and absolute magnitudes of $H_{r}=6.2-10.8 \mathrm{mag}$ (as calculated from our light-curve observations). This sample far outnumbers (by a factor of 2.5) previously reported variability studies for faint $\left(H_{r} \geqslant 8 \mathrm{mag}\right)$ TNOs. Our sample thus much more thoroughly probes a smaller size regime than previous light-curve studies have typically studied, thanks to the combined aperture size and field of view of Subaru/HSC. Information about the individual objects in our sample can be found in Table 2 .

Data were collected by M. Alexandersen and S.-Y. Wang on the nights of 2016 August 25 and 26, using HSC on Subaru Telescope, located on Maunakea, Hawaii, USA. ${ }^{14}$ Figure 1 shows our field locations in relation to the swarm of OSSOS TNOs. As TNOs are typically brightest in the $r$ band, this filter was used. The six fields were observed in a repeated sequence with one $300 \mathrm{~s}$ exposure of each field, resulting in about 36 minutes between repeat observations of the same field. Because the observing nights were approximately 1.5 months from opposition, we were unable to observe our fields for the entire night; the four fields closer to opposition thus were observed 12 times each night, while two other fields were observed 10 times each night. Observations were taken at air masses ranging from 1.02 to 2.04. The conditions were generally good (transparency near 1.0, with the seeing mostly between 0 " 65 and 1."0), apart from about one hour of clouds and very low transparency on the second night. One or two observations of each field were, therefore, unusable for this project.

\footnotetext{
14 All data obtained as part of this project can be downloaded from https:/ smoka.nao.ac.jp/fssearch or http://www.cadc-ccda.hia-iha.nrc-cnrc.gc.ca/en/ search/.
} 


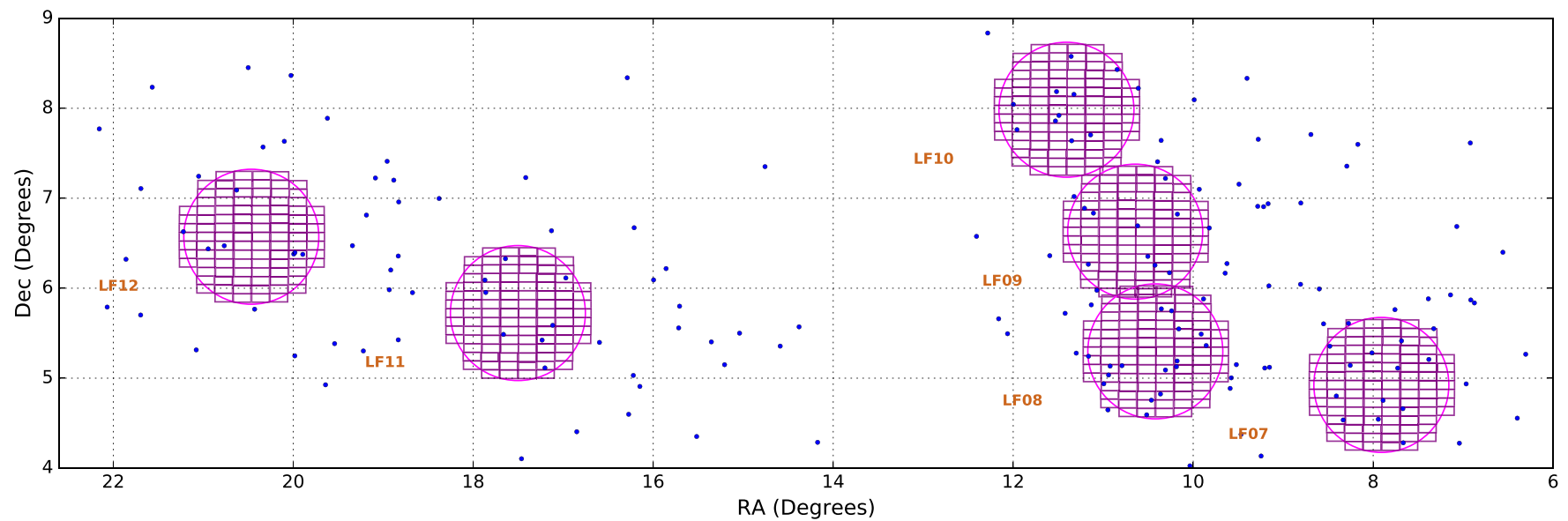

Figure 1. Location of our 2016 fields relative to TNOs discovered in the OSSOS L and S blocks (left and right, respectively). The large field of view of HSC (magenta) allowed us to observe many objects at once. The field centers used are listed in Table 1.

\section{Photometry and Calibration}

Images were processed using hscPipe, ${ }^{15}$ the official HSC data reduction software package, which includes calibration of astrometry/photometry and basic image processing (bias, darks, and flats; Bosch et al. 2018). hscPipe was developed as a prototype pipeline for the Large Synoptic Survey Telescope (LSST) project (Ivezic et al. 2019). The algorithms and conceptions of hscPipe were inherited from the Sloan Digital Sky Survey (SDSS) Photo Pipeline (Lupton et al. 2001). The standard outputs of the hscPipe process include detection catalogs and calibrated images. We used the PanSTARRS1 PV2 catalog (PS1; Schlafly et al. 2012; Tonry et al. 2012; Magnier et al. 2013) as the reference catalog for photometry and astrometry.

Photometry of bright point sources revealed systematic variability (of the order of $0.01 \mathrm{mag}$ ), caused by the fact that hscPipe calibrates images individually; images of the same field can thus have slight discrepancies relative to each other due to uncertainties in the zero-point determinations. To mitigate for this effect, background stars were inspected to find a set of 10-15 nonvariable stars, defined as those that exhibit similar systematic variability, to reveal the systematic variability of the zero-point error. A secondary correction was then applied to the zero-points, such that the average magnitude of the nonvariable stars is constant across the set of images. This adjustment was typically smaller than $0.02 \mathrm{mag}$ but is important for obtaining the most accurate TNO photometry possible.

In order to get the best possible photometric measurements of our TNOs, we used the Python package TRIPPy (Fraser et al. 2016). TNOs move at typical rates of $3^{\prime \prime}-6^{\prime \prime} / \mathrm{hr}$ at opposition, thus moving up to 0.15 during our $300 \mathrm{~s}$ exposures, causing slight trailing. TRIPPy uses knowledge of the rate and angle of motion to accurately measure photometry for moving objects using an elongated aperture and appropriate aperture correction.

We obtained 9-23 useful measurements for each object over two nights; the median number of measurements per object is 19 . One or two images per field were discarded due to low transparency during one hour on the second night; while some reduced transparency can be corrected for in calibration, we found that images with transparency $<50 \%$ consistently produced anomalous results, so we chose to exclude those images. Many objects lost one

\footnotetext{
${ }^{15}$ https://hscdata.mtk.nao.ac.jp:4443/hsc_bin_dist/index.html
}

or more measurement due to being too close to a background source for reliable photometry. Three objects $\left(2015 \mathrm{RE}_{280}, 2000\right.$ $\mathrm{QH}_{226}$, and $2015 \mathrm{RR}_{278}$ ) only have data on one night because they fell into chip gaps on one of the two nights; this was difficult to avoid when optimizing field location for up to 17 objects moving at different rates. One object $\left(2015 \mathrm{RZ}_{279}\right)$ was on a different chip on the two nights; however, we are confident that our zero-point calibration is accurate such that there is not an offset between the two nights. The final calibrated photometric measurements as well as the calibrated zero-points can be found in Table 3.

\section{Results}

We measured partial light curves for 63 TNOs and two Centaurs. The measurements can be found in graphical form in Figures 2-6; the photometric data can be found in Table 3. Several objects (such as $2013 \mathrm{UL}_{15}, 2015 \mathrm{RO}_{281}, 2003 \mathrm{SP}_{317}, 2015 \mathrm{RB}_{280}$, and $2013 \mathrm{UN}_{15}$ ) feature clear, high-amplitude variability. We investigated the relationships between our measured light-curve variability and other properties, such as the absolute magnitude, dynamical class, and other orbital parameters (Section 4.1). For the best quality light curves, estimates of the rotation periods were also made (Section 4.2).

In order to estimate the uncertainty on parameters, such as the magnitude variation, period, correlation coefficient, and so on, a resampling method was used. One thousand resampled data sets were generated by resampling each magnitude measurement of each object. This resampling was drawn from a normal distribution centered on the measured value with a width equal to the measurement uncertainty. The analysis was then performed on each resampled data set independently to find the distribution of resultant values. The $2 \sigma$ uncertainty on a value is then taken to be the range that spans the central $95 \%$ of values (i.e., $2.5 \%$ in each tail).

\subsection{Amplitude Analysis}

The mean rotational period of TNOs has been found to be 7-9 hr (Duffard et al. 2009; Benecchi \& Sheppard 2013; Thirouin et al. 2016), although periods range from $3.9 \mathrm{hr}$ (Haumea) to $154 \mathrm{hr}$ (Pluto). While we do not have full light curves for all of our target objects, the mean period of 7-9 hr means that we likely observed a near-maximum and nearminimum of most of our target objects. Simple simulations of 
Table 2

Summary of Information about the Objects in our Sample

\begin{tabular}{|c|c|c|c|c|c|c|c|c|c|c|}
\hline ID & Field & $a(\mathrm{au})$ & $e$ & $i(\mathrm{deg})$ & Class & MPC & $m_{r}(\mathrm{mag})$ & $H_{r}(\mathrm{mag})$ & $\Delta_{\mathrm{mag}}$ & $\sigma_{\mathrm{mag}}$ \\
\hline o5t09PD & LF09 & $67.760 \pm 0.009$ & 0.459 & 3.575 & det & $2014 \mathrm{UA}_{225}$ & $22.627 \pm 0.002$ & 7.033 & $0.110_{-0.012}^{+0.012}$ & $0.027_{-0.002}^{+0.002}$ \\
\hline o3143 & LF11 & $45.785 \pm 0.004$ & 0.097 & 2.025 & cla & $2013 \mathrm{UL}_{15}$ & $22.989 \pm 0.002$ & 6.702 & $0.363_{-0.011}^{+0.012}$ & $0.109_{-0.002}^{+0.002}$ \\
\hline $\mathrm{o} 5 \mathrm{~s} 21$ & LF10 & $43.851 \pm 0.007$ & 0.170 & 2.373 & cla & $2015 \mathrm{RO}_{281}$ & $23.225 \pm 0.003$ & 7.564 & $0.36_{-0.02}^{+0.02}$ & $0.106_{-0.003}^{+0.003}$ \\
\hline o3157 & LF12 & $45.044 \pm 0.004$ & 0.075 & 1.841 & res $11: 6$ & $2013 \mathrm{UM}_{15}$ & $23.297 \pm 0.003$ & 6.872 & $0.127_{-0.014}^{+0.015}$ & $0.034_{-0.003}^{+0.003}$ \\
\hline $\mathrm{o} 5 \mathrm{t} 03$ & LF09 & $25.967 \pm 0.0014$ & 0.288 & 18.849 & cen & $2015 \mathrm{RD}_{277}$ & $23.380 \pm 0.003$ & 10.825 & $0.15_{-0.02}^{+0.03}$ & $0.041_{-0.004}^{+0.004}$ \\
\hline o5t34PD & LF07 & $45.959 \pm 0.003$ & 0.170 & 5.081 & res $17: 9$ & $2003 \mathrm{SP}_{317}$ & $23.511 \pm 0.006$ & 7.226 & $0.56_{-0.04}^{+0.05}$ & $0.177_{-0.006}^{+0.007}$ \\
\hline o5s 07 & LF08 & $39.332 \pm 0.004$ & 0.249 & 16.270 & res $3: 2$ & $2015 \mathrm{RU}_{277}$ & $23.615 \pm 0.004$ & 8.957 & $0.22_{-0.02}^{+0.02}$ & $0.048_{-0.003}^{+0.004}$ \\
\hline o5s52 & LF10 & $50.85 \pm 0.03$ & 0.247 & 27.196 & res $11: 5$ & $2015 \mathrm{RU}_{278}$ & $23.616 \pm 0.003$ & 7.054 & $0.314_{-0.014}^{+0.014}$ & $0.094_{-0.003}^{+0.003}$ \\
\hline $05 \mathrm{~s} 36$ & LF09 & $43.258 \pm 0.003$ & 0.053 & 2.206 & cla & $2015 \mathrm{RB}_{281}$ & $23.638 \pm 0.003$ & 7.569 & $0.43_{-0.02}^{+0.02}$ & $0.128_{-0.004}^{+0.004}$ \\
\hline o5s 05 & LF10 & $21.981 \pm 0.010$ & 0.479 & 15.389 & cen & $2015 \mathrm{RV}_{245}$ & $23.640 \pm 0.004$ & 10.767 & $0.10_{-0.02}^{+0.02}$ & $0.025_{-0.004}^{+0.004}$ \\
\hline o3164 & LF11 & $44.355 \pm 0.003$ & 0.073 & 1.972 & cla & $2013 \mathrm{UW}_{16}$ & $23.729 \pm 0.004$ & 7.233 & $0.13_{-0.02}^{+0.02}$ & $0.038_{-0.004}^{+0.004}$ \\
\hline o3146 & LF12 & $46.612 \pm 0.004$ & 0.079 & 2.471 & cla & $2013 \mathrm{UP}_{15}$ & $23.730 \pm 0.004$ & 7.404 & $0.29_{-0.02}^{+0.02}$ & $0.089_{-0.004}^{+0.004}$ \\
\hline o5t08 & LF08 & $38.848 \pm 0.003$ & 0.057 & 1.097 & cla & $2015 \mathrm{RY}_{278}$ & $23.751 \pm 0.004$ & 8.164 & $0.21_{-0.02}^{+0.02}$ & $0.057_{-0.004}^{+0.004}$ \\
\hline $\mathrm{o} 5 \mathrm{t} 30$ & LF08 & $43.791 \pm 0.008$ & 0.073 & 2.170 & cla & $2015 \mathrm{RB}_{280}$ & $23.811 \pm 0.004$ & 7.648 & $0.55_{-0.02}^{+0.02}$ & $0.159_{-0.005}^{+0.005}$ \\
\hline$o 5 t 42$ & LF07 & $39.451 \pm 0.011$ & 0.204 & 1.587 & res $3: 2$ & $2000 \mathrm{QA}_{243}$ & $23.833 \pm 0.004$ & 7.254 & $0.16_{-0.02}^{+0.02}$ & $0.045_{-0.004}^{+0.004}$ \\
\hline o5s 14 & LF07 & $34.841 \pm 0.002$ & 0.047 & 7.122 & res $5: 4$ & $2015 \mathrm{RN}_{278}$ & $23.911 \pm 0.004$ & 8.488 & $0.22_{-0.02}^{+0.02}$ & $0.054_{-0.004}^{+0.004}$ \\
\hline $\mathrm{o} 5 \mathrm{t} 51$ & LF08 & $59.83 \pm 0.10$ & 0.425 & 13.851 & res $14: 5$ & $2015 \mathrm{RG}_{279}$ & $23.936 \pm 0.004$ & 6.833 & $0.24_{-0.02}^{+0.03}$ & $0.057_{-0.004}^{+0.004}$ \\
\hline o3182 & LF11 & $43.657 \pm 0.005$ & 0.286 & 24.987 & res $7: 4$ & $2013 \mathrm{UK}_{17}$ & $23.936 \pm 0.005$ & 6.621 & $0.15_{-0.02}^{+0.02}$ & $0.044_{-0.005}^{+0.006}$ \\
\hline o3163 & LF12 & $45.132 \pm 0.004$ & 0.054 & 3.363 & cla & $2013 \mathrm{UN}_{15}$ & $23.986 \pm 0.005$ & 7.493 & $0.56_{-0.03}^{+0.03}$ & $0.161_{-0.004}^{+0.005}$ \\
\hline o3183 & LF11 & $200.2 \pm 0.5$ & 0.781 & 10.652 & det & $2013 \mathrm{UT}_{15}$ & $24.001 \pm 0.005$ & 6.168 & $0.33_{-0.02}^{+0.02}$ & $0.098_{-0.005}^{+0.005}$ \\
\hline o5s 35 & LF09 & $46.40 \pm 0.02$ & 0.161 & 24.307 & cla & $2015 \mathrm{RC}_{277}$ & $24.065 \pm 0.005$ & 8.023 & $0.37_{-0.03}^{+0.03}$ & $0.106_{-0.005}^{+0.005}$ \\
\hline 03155 & LF11 & $44.004 \pm 0.004$ & 0.096 & 2.358 & cla & 2013 UY $_{16}$ & $24.086 \pm 0.005$ & 7.667 & $0.37_{-0.02}^{+0.02}$ & $0.121_{-0.005}^{+0.005}$ \\
\hline o5t16 & LF08 & $44.79 \pm 0.02$ & 0.176 & 17.756 & cla & $2015 \mathrm{RY}_{279}$ & $24.158 \pm 0.005$ & 8.358 & $0.36_{-0.03}^{+0.04}$ & $0.093_{-0.006}^{+0.006}$ \\
\hline $05 \mathrm{~s} 30$ & LF07 & $41.477 \pm 0.005$ & 0.073 & 33.327 & cla & $2015 \mathrm{RR}_{280}$ & $24.164 \pm 0.007$ & 8.247 & $0.27_{-0.03}^{+0.03}$ & $0.063_{-0.006}^{+0.006}$ \\
\hline o3171 & LF12 & $45.319 \pm 0.003$ & 0.014 & 1.927 & cla & $2013 \mathrm{UW}_{17}$ & $24.178 \pm 0.006$ & 7.612 & $0.42_{-0.03}^{+0.03}$ & $0.125_{-0.005}^{+0.006}$ \\
\hline o5t06 & LF08 & $72.06 \pm 0.03$ & 0.535 & 12.327 & sca & $2015 \mathrm{RZ}_{278}$ & $24.183 \pm 0.006$ & 8.941 & $0.25_{-0.03}^{+0.03}$ & $0.069_{-0.006}^{+0.006}$ \\
\hline o5s 22 & LF10 & $46.162 \pm 0.010$ & 0.222 & 8.482 & res $19: 10$ & $2015 \mathrm{RG}_{281}$ & $24.207 \pm 0.005$ & 8.538 & $0.21_{-0.02}^{+0.02}$ & $0.059_{-0.005}^{+0.005}$ \\
\hline $05 \mathrm{~s} 15$ & LF07 & $45.439 \pm 0.009$ & 0.238 & 25.362 & res $13: 7$ & $2015 \mathrm{RO}_{278}$ & $24.246 \pm 0.006$ & 8.809 & $0.31_{-0.03}^{+0.03}$ & $0.084_{-0.006}^{+0.006}$ \\
\hline 03165 & LF11 & $44.609 \pm 0.007$ & 0.278 & 11.207 & sca & $2013 \mathrm{UZ}_{16}$ & $24.272 \pm 0.006$ & 7.775 & $0.36_{-0.03}^{+0.03}$ & $0.095_{-0.007}^{+0.007}$ \\
\hline 03120 & LF12 & $39.425 \pm 0.010$ & 0.181 & 2.085 & res $3: 2$ & $2013 \mathrm{UV}_{17}$ & $24.335 \pm 0.008$ & 8.354 & $0.69_{-0.04}^{+0.04}$ & $0.185_{-0.008}^{+0.008}$ \\
\hline o5s 19 & LF10 & $45.054 \pm 0.006$ & 0.194 & 17.960 & res $11: 6$ & $2015 \mathrm{RT}_{278}$ & $24.357 \pm 0.006$ & 8.758 & $0.38_{-0.03}^{+0.03}$ & $0.105_{-0.006}^{+0.006}$ \\
\hline $\mathrm{o} 5 \mathrm{t} 10$ & LF07 & $37.844 \pm 0.006$ & 0.051 & 1.540 & cla & $2015 \mathrm{RW}_{278}$ & $24.375 \pm 0.007$ & 8.768 & $0.31_{-0.04}^{+0.05}$ & $0.081_{-0.008}^{+0.008}$ \\
\hline o5t44 & LF08 & $43.560 \pm 0.004$ & 0.071 & 3.566 & cla & $2015 \mathrm{RZ}_{279}$ & $24.383 \pm 0.007$ & 7.768 & $0.28_{-0.03}^{+0.03}$ & $0.085_{-0.006}^{+0.005}$ \\
\hline 03162 & LF11 & $39.230 \pm 0.004$ & 0.238 & 4.416 & res $3: 2$ & $2013 \mathrm{UX}_{16}$ & $24.415 \pm 0.008$ & 7.950 & $0.27_{-0.04}^{+0.05}$ & $0.076_{-0.010}^{+0.010}$ \\
\hline o5s 24 & LF10 & $50.376 \pm 0.018$ & 0.278 & 9.376 & res $13: 6$ & $2015 \mathrm{RV}_{278}$ & $24.435 \pm 0.007$ & 8.657 & $0.58_{-0.04}^{+0.04}$ & $0.153_{-0.007}^{+0.007}$ \\
\hline o3119 & LF11 & $45.334 \pm 0.003$ & 0.126 & 1.480 & cla & $2013 \mathrm{SM}_{100}$ & $24.447 \pm 0.007$ & 8.507 & $0.68_{-0.04}^{+0.04}$ & $0.174_{-0.007}^{+0.007}$ \\
\hline $05 s 42$ & LF10 & $39.323 \pm 0.011$ & 0.165 & 5.772 & res $3: 2$ & $2015 \mathrm{RV}_{277}$ & $24.482 \pm 0.007$ & 8.284 & $0.38_{-0.04}^{+0.05}$ & $0.102_{-0.010}^{+0.009}$ \\
\hline $\mathrm{o} 5 \mathrm{t} 27$ & LF08 & $43.907 \pm 0.011$ & 0.079 & 1.498 & cla & $2015 \mathrm{RW}_{279}$ & $24.490 \pm 0.007$ & 8.348 & $0.42_{-0.04}^{+0.04}$ & $0.101_{-0.006}^{+0.006}$ \\
\hline o5t48 & LF07 & $42.183 \pm 0.003$ & 0.156 & 5.209 & res $5: 3$ & $2015 \mathrm{RJ}_{278}$ & $24.526 \pm 0.011$ & 7.693 & $0.41_{-0.04}^{+0.04}$ & $0.107_{-0.009}^{+0.009}$ \\
\hline o5t13 & LF08 & $79.06 \pm 0.03$ & 0.535 & 17.629 & res $17: 4$ & $2015 \mathrm{RL}_{278}$ & $24.531 \pm 0.007$ & 8.849 & $0.41_{-0.05}^{+0.05}$ & $0.100_{-0.008}^{+0.008}$ \\
\hline $\mathrm{o} 5 \mathrm{t} 32$ & LF08 & $43.791 \pm 0.005$ & 0.035 & 2.209 & cla & $2015 \mathrm{RT}_{279}$ & $24.537 \pm 0.008$ & 8.292 & $0.48_{-0.05}^{+0.05}$ & $0.117_{-0.010}^{+0.010}$ \\
\hline o3184 & LF11 & $44.012 \pm 0.003$ & 0.082 & 1.435 & cla & $2013 \mathrm{UR}_{22}$ & $24.547 \pm 0.009$ & 7.858 & $0.44_{-0.06}^{+0.06}$ & $0.117_{-0.010}^{+0.011}$ \\
\hline o3144 & LF12 & $42.793 \pm 0.005$ & 0.042 & 2.940 & cla & $2013 \mathrm{UC}_{18}$ & $24.557 \pm 0.008$ & 8.261 & $0.47_{-0.04}^{+0.04}$ & $0.141_{-0.009}^{+0.010}$ \\
\hline uo3188 & LF11 & $43.935 \pm 0.004$ & 0.081 & 2.479 & cla & $2013 \mathrm{ST}_{102}$ & $24.558 \pm 0.007$ & 8.275 & $0.53_{-0.05}^{+0.04}$ & $0.169_{-0.008}^{+0.009}$ \\
\hline $\mathrm{o} 5 \mathrm{t} 07$ & LF07 & $39.513 \pm 0.009$ & 0.192 & 2.802 & res $3: 2$ & $2000 \mathrm{QH}_{226}$ & $24.611 \pm 0.014$ & 9.159 & $0.48_{-0.06}^{+0.06}$ & $0.143_{-0.014}^{+0.015}$ \\
\hline o5t41 & LF08 & $44.310 \pm 0.004$ & 0.043 & 0.997 & cla & $2015 \mathrm{RE}_{280}$ & $24.659 \pm 0.011$ & 8.085 & $0.23_{-0.04}^{+0.05}$ & $0.068_{-0.009}^{+0.012}$ \\
\hline $\mathrm{o} 5 \mathrm{t} 22$ & LF08 & $39.58 \pm 0.02$ & 0.176 & 5.457 & res $3: 2$ & $2015 \mathrm{RT}_{277}$ & $24.664 \pm 0.009$ & 8.612 & $0.28_{-0.03}^{+0.05}$ & $0.076_{-0.008}^{+0.009}$ \\
\hline o5t49 & LF08 & $44.851 \pm 0.004$ & 0.096 & 1.968 & cla & $2015 \mathrm{RA}_{280}$ & $24.672 \pm 0.008$ & 7.807 & $0.64_{-0.04}^{+0.04}$ & $0.204_{-0.008}^{+0.008}$ \\
\hline o5s61 & LF10 & $44.942 \pm 0.012$ & 0.117 & 3.023 & cla & $2015 \mathrm{RP}_{281}$ & $24.696 \pm 0.009$ & 7.879 & $0.65_{-0.04}^{+0.05}$ & $0.177_{-0.009}^{+0.009}$ \\
\hline $05 t 23$ & LF08 & $46.215 \pm 0.007$ & 0.132 & 5.089 & cla & $2015 \mathrm{RU}_{279}$ & $24.813 \pm 0.009$ & 8.760 & $0.66_{-0.06}^{+0.08}$ & $0.174_{-0.012}^{+0.012}$ \\
\hline $05 s 40$ & LF09 & $44.310 \pm 0.007$ & 0.075 & 2.276 & cla & $2015 \mathrm{RK}_{281}$ & $24.965 \pm 0.010$ & 8.702 & $0.41_{-0.04}^{+0.05}$ & $0.117_{-0.009}^{+0.009}$ \\
\hline $05 \mathrm{t} 26$ & LF09 & $43.173 \pm 0.004$ & 0.043 & 16.024 & cla & $2015 \mathrm{RX}_{279}$ & $24.973 \pm 0.013$ & 8.855 & $0.41_{-0.05}^{+0.07}$ & $0.115_{-0.014}^{+0.014}$ \\
\hline uo5t55 & LF08 & $44.25 \pm 0.02$ & 0.073 & 1.544 & cla & $2015 \mathrm{RR}_{281}$ & $25.015 \pm 0.011$ & 8.702 & $0.54_{-0.07}^{+0.08}$ & $0.129_{-0.013}^{+0.013}$ \\
\hline o5s 29 & LF07 & $41.396 \pm 0.003$ & 0.045 & 34.929 & cla & $2015 \mathrm{RO}_{280}$ & $25.023 \pm 0.012$ & 9.111 & $0.33_{-0.04}^{+0.05}$ & $0.097_{-0.011}^{+0.011}$ \\
\hline $05 s 43$ & LF07 & $70.84 \pm 0.07$ & 0.465 & 10.192 & $\operatorname{det}$ & $2015 \mathrm{RH}_{279}$ & $25.044 \pm 0.011$ & 8.837 & $0.49_{-0.08}^{+0.07}$ & $0.120_{-0.015}^{+0.014}$ \\
\hline $\mathrm{o} 5 \mathrm{t} 25$ & LF08 & $43.288 \pm 0.006$ & 0.047 & 0.461 & cla & $2015 \mathrm{RC}_{280}$ & $25.084 \pm 0.012$ & 8.971 & $0.40_{-0.06}^{+0.06}$ & $0.099_{-0.011}^{+0.011}$ \\
\hline $\mathrm{o} 5 \mathrm{t} 21$ & LF08 & $43.633 \pm 0.003$ & 0.074 & 6.872 & res $7: 4$ & $2015 \mathrm{RE}_{278}$ & $25.104 \pm 0.014$ & 9.082 & $0.59_{-0.06}^{+0.07}$ & $0.165_{-0.013}^{+0.013}$ \\
\hline o5s55 & LF09 & $46.93 \pm 0.02$ & 0.173 & 4.812 & cla & $2015 \mathrm{RH}_{281}$ & $25.107 \pm 0.010$ & 8.528 & $0.59_{-0.06}^{+0.07}$ & $0.148_{-0.010}^{+0.011}$ \\
\hline
\end{tabular}


Table 2

(Continued)

\begin{tabular}{lccccllcrcr}
\hline \hline ID & Field & $a(\mathrm{au})$ & $e$ & $i(\mathrm{deg})$ & Class & MPC & $m_{r}(\mathrm{mag})$ & $H_{r}(\mathrm{mag})$ & $\Delta_{\text {mag }}$ \\
\hline o5s26 & LF09 & $41.344 \pm 0.007$ & 0.112 & 17.483 & cla & $2015 \mathrm{RD}_{281}$ & $25.111 \pm 0.014$ & 9.299 & $0.54_{-0.05}^{+0.05}$ & $0.152_{-0.012}^{+0.012}$ \\
o5s12 & LF10 & $34.791 \pm 0.005$ & 0.100 & 2.668 & res 5:4 & $2015 \mathrm{RR}_{278}$ & $25.118 \pm 0.015$ & 9.798 & $0.40_{-0.06}^{+0.08}$ & $0.13_{-0.02}^{+0.02}$ \\
o5s49 & LF07 & $44.017 \pm 0.007$ & 0.044 & 2.088 & cla & $2015 \mathrm{RQ}_{280}$ & $25.178 \pm 0.012$ & 8.889 & $1.01_{-0.09}^{+0.10}$ & $0.22_{-0.02}^{+0.02}$ \\
o5s60 & LF10 & $42.530 \pm 0.007$ & 0.128 & 40.362 & cla & $2015 \mathrm{RN}_{281}$ & $25.180 \pm 0.012$ & 8.510 & $0.51_{-0.06}^{+0.07}$ & $0.127_{-0.012}^{+0.012}$ \\
o5t24 & LF09 & $44.953 \pm 0.006$ & 0.107 & 2.398 & cla & $2015 \mathrm{RH}_{280}$ & $25.257 \pm 0.014$ & 9.199 & $0.80_{-0.08}^{+0.08}$ & $0.20_{-0.02}^{+0.02}$ \\
05s57 & LF07 & $41.356 \pm 0.011$ & 0.140 & 32.358 & cla & $2015 \mathrm{RW}_{280}$ & $25.276 \pm 0.016$ & 8.649 & $0.84_{-0.09}^{+0.10}$ & $0.227_{-0.015}^{+0.016}$ \\
uo5s77 & LF09 & $65.95 \pm 0.11$ & 0.413 & 38.607 & res 13:4 & $2015 \mathrm{RS}_{281}$ & $25.604 \pm 0.018$ & 9.477 & $0.98_{-0.09}^{+0.09}$ & $0.295_{-0.02}^{+0.02}$ \\
\hline
\end{tabular}

Note. ID refers to the internal OSSOS designation (as used in (Bannister et al. 2018)). Field is the field in which the target was observed (Table 1). $a, e$ and $i$ are the barycentric orbital parameters semi-major axis, eccentricity and inclination (uncertainties on eccentricity and inclination are always 0.001 or smaller and are therefore not listed here). Class is the dynamical classification of the object. MPC is the Minor Planet Center (https://minorplanetcenter.net/iau/mpc.html) designation (Note: all objects will have MPC designations before the publication of this work). $m_{r}$ and $H_{r}$ are the mean apparent and absolute magnitudes of the objects, as measured in this work (these are the magnitudes used everywhere in this paper, as they are more accurate than the discovery magnitudes from OSSOS); due to the quality of the OSSOS orbits, the uncertainty on $H_{r}$ is identical to that of $m_{r}$ to the given precision. $\Delta_{\text {mag }}$ is the maximum magnitude variation measured for the object within this work. $\sigma_{\mathrm{mag}}$ is the standard deviation of the magnitudes measured in this work. This table is ordered in the same order as Figures 3 to 6: brightest object at the top to faintest object at the bottom.

(This table is available in machine-readable form.)

Table 3

Photometric Measurements from This HSC Data Set

\begin{tabular}{lcc}
\hline \hline MJD-57620 & $m_{r} \pm \sigma_{\text {Rand }}$ & $Z p \pm \sigma_{\text {Sys }}$ \\
\hline & $03119,2013 \mathrm{SM}_{100}$ & \\
6.3913125 & $24.09 \pm 0.03$ & $26.995 \pm 0.005$ \\
6.4154337 & $24.21 \pm 0.02$ & $27.012 \pm 0.005$ \\
6.4393438 & $24.45 \pm 0.02$ & $27.022 \pm 0.005$ \\
6.4634385 & $24.69 \pm 0.04$ & $27.058 \pm 0.005$ \\
6.4876346 & $24.78 \pm 0.03$ & $27.047 \pm 0.005$ \\
6.5123402 & $24.56 \pm 0.03$ & $27.039 \pm 0.005$ \\
6.5375195 & $24.41 \pm 0.03$ & $27.043 \pm 0.005$ \\
6.5628085 & $24.29 \pm 0.03$ & $27.045 \pm 0.005$ \\
6.5875091 & $24.28 \pm 0.02$ & $27.038 \pm 0.005$ \\
7.3876072 & $24.55 \pm 0.03$ & $26.978 \pm 0.005$ \\
7.4114040 & $24.67 \pm 0.03$ & $27.002 \pm 0.005$ \\
7.4353624 & $24.45 \pm 0.02$ & $26.885 \pm 0.005$ \\
7.4595078 & $24.47 \pm 0.02$ & $26.869 \pm 0.005$ \\
7.5081300 & $24.36 \pm 0.03$ & $26.497 \pm 0.005$ \\
7.5584662 & $24.35 \pm 0.02$ & $27.019 \pm 0.005$ \\
7.5830743 & $24.42 \pm 0.02$ & $27.004 \pm 0.005$ \\
7.6079518 & $24.58 \pm 0.02$ & $26.994 \pm 0.005$ \\
\hline
\end{tabular}

Note. $\sigma_{\text {Rand }}$ is the random uncertainty of the $m_{r}$ magnitude measurement. $Z p$ is the zero-point used. $\sigma_{\mathrm{Sys}}$ is the systematic uncertainty on the zero-point (and thus also on the measurement). This is an example for a single object; all measurements from all 65 objects are available in machine readable format.

(This table is available in its entirety in machine-readable form.)

our observations confirm that for objects of a given period between 3 and $40 \mathrm{hr}$, we observe more than $80 \%$ of the variability on average (averaged over the random starting phase); for periods between 3 and $22 \mathrm{hr}$, we observe more than $88 \%$ of the variability on average. It is thus reasonable to assume that our measurements of $\Delta_{\text {mag }}$ (the maximum minus the minimum magnitude) are close to the full light-curve amplitudes and that the slight underestimation will not affect our results (such as whether or not correlations exist). In this subsection, we removed the two Centaurs $\left(2015 \mathrm{RV}_{245}\right.$ and $2015 \mathrm{RD}_{277}$ ) from the sample, because the surface and rotation of these objects might have been altered by close approaches to the Sun or planets (Duffard et al. 2009; Thirouin et al. 2010). As an alternative to the difference between the brightest and faintest observation $\left(\Delta_{\mathrm{mag}}\right)$, we also examine the standard deviation $\left(\sigma_{\mathrm{mag}}\right)$ of all the observations of each object, because the standard deviation is a measure of overall variation that is less influenced by individual anomalous measurements.

In Figure 7, we plot both $\Delta_{\text {mag }}$ and $\sigma_{\text {mag }}$ versus object $H_{r}$, semimajor axis $a$, and inclination $i$ to illustrate possible relations between these parameters. In a sample of 128 objects, Benecchi \& Sheppard (2013) found a statistically significant correlation ( $\rho=0.288$ and $P=0.001$ ) between the light-curve amplitude and absolute magnitude. Their sample spanned absolute magnitudes from 0 to $12 \mathrm{mag}$, although most were between 4 and $7 \mathrm{mag}$; very few had absolute magnitudes fainter than 8 mag. Our sample of 63 TNOs span $H_{r}=6.2-10.8 \mathrm{mag},{ }^{16}$ with more than half the sample at $H_{r}>8.0 \mathrm{mag}$; our sample thus represents variability properties for fainter TNOs than the bulk of the Benecchi \& Sheppard (2013) sample. Using the Spearman Rank correlation test (Spearman 1904) and our independent sample of $63 \mathrm{TNOs}$, we find $\rho=0.37$ and $P=0.003$ for $\Delta_{\operatorname{mag}}$ versus $H_{r}$ and $\rho=0.31$ and $P=0.013$ for $\sigma_{\mathrm{mag}}$ versus $H_{r}$. Our data thus strongly support the existence of a weak relationship where intrinsically fainter (likely smaller) bodies, on average, have larger light-curve amplitudes. This is likely due to increasingly elongated shapes. Benecchi \& Sheppard (2013) also found a borderline significant anticorrelation $(\rho=-0.170$ and $P=0.056)$ between the lightcurve amplitude and inclination (i); however, our data do not support the existence of such an anticorrelation.

Because the cold classicals are thought to have formed in place while all the hot populations (hot classicals, resonant, detached, and scattering) are thought to have been scattered outward to some extent after having formed closer to the Sun (e.g., Levison et al. 2008; Li et al. 2008; Batygin et al. 2011), we also test the dynamically cold and hot samples separately to identify whether a trend might be present in one population and

\footnotetext{
${ }^{16}$ Whenever we discuss apparent and absolute magnitudes for our sample objects, those magnitudes are measured/calculated from our light curves observations, as these are more accurate than the discovery magnitudes reported by OSSOS.
} 

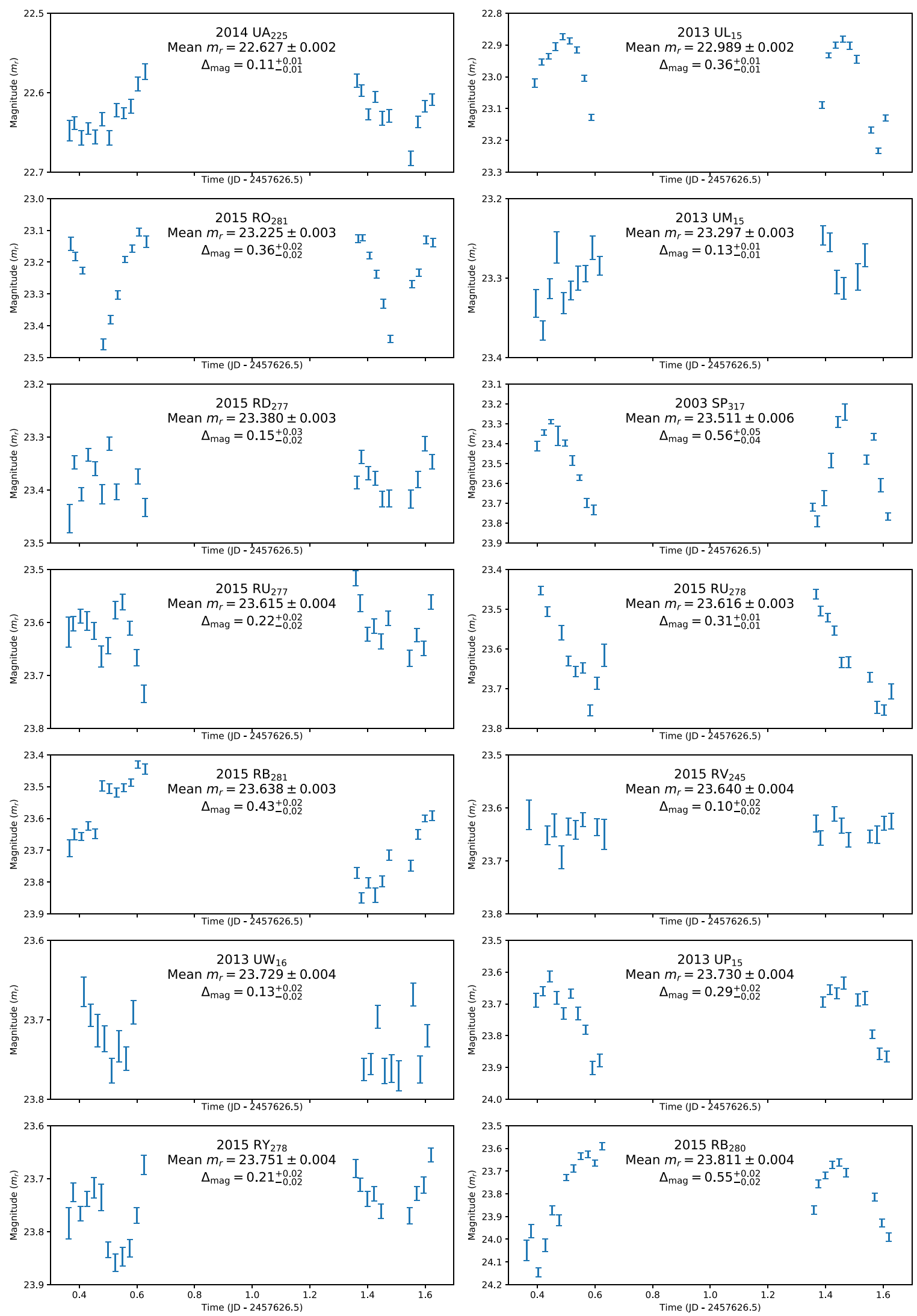

Figure 2. Absolutely calibrated photometry of our TNO sample. Objects $0-13$, ordered by their average magnitude. 

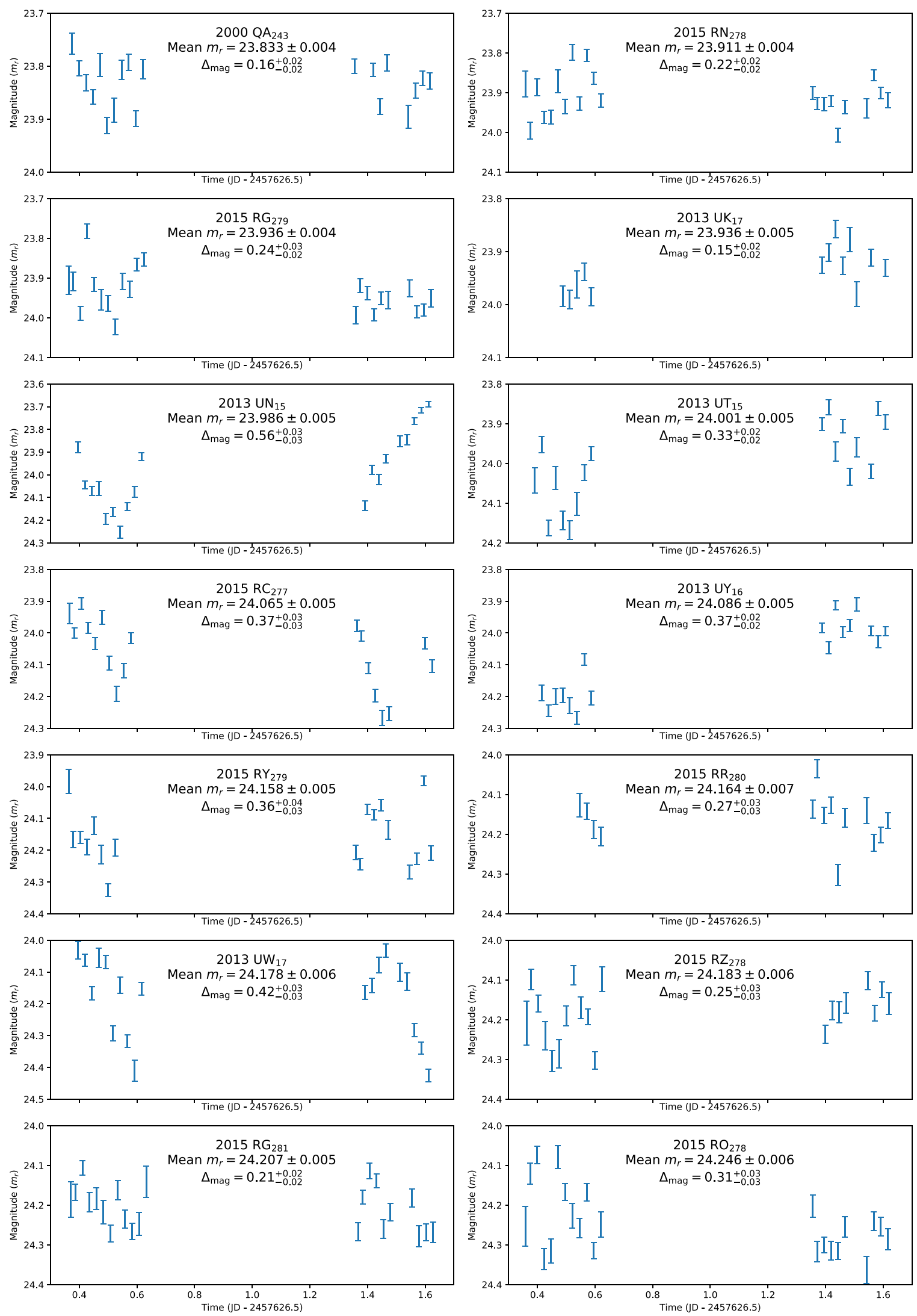

Figure 3. Absolutely calibrated photometry of our TNO sample. Objects $14-27$, ordered by their average magnitude. 

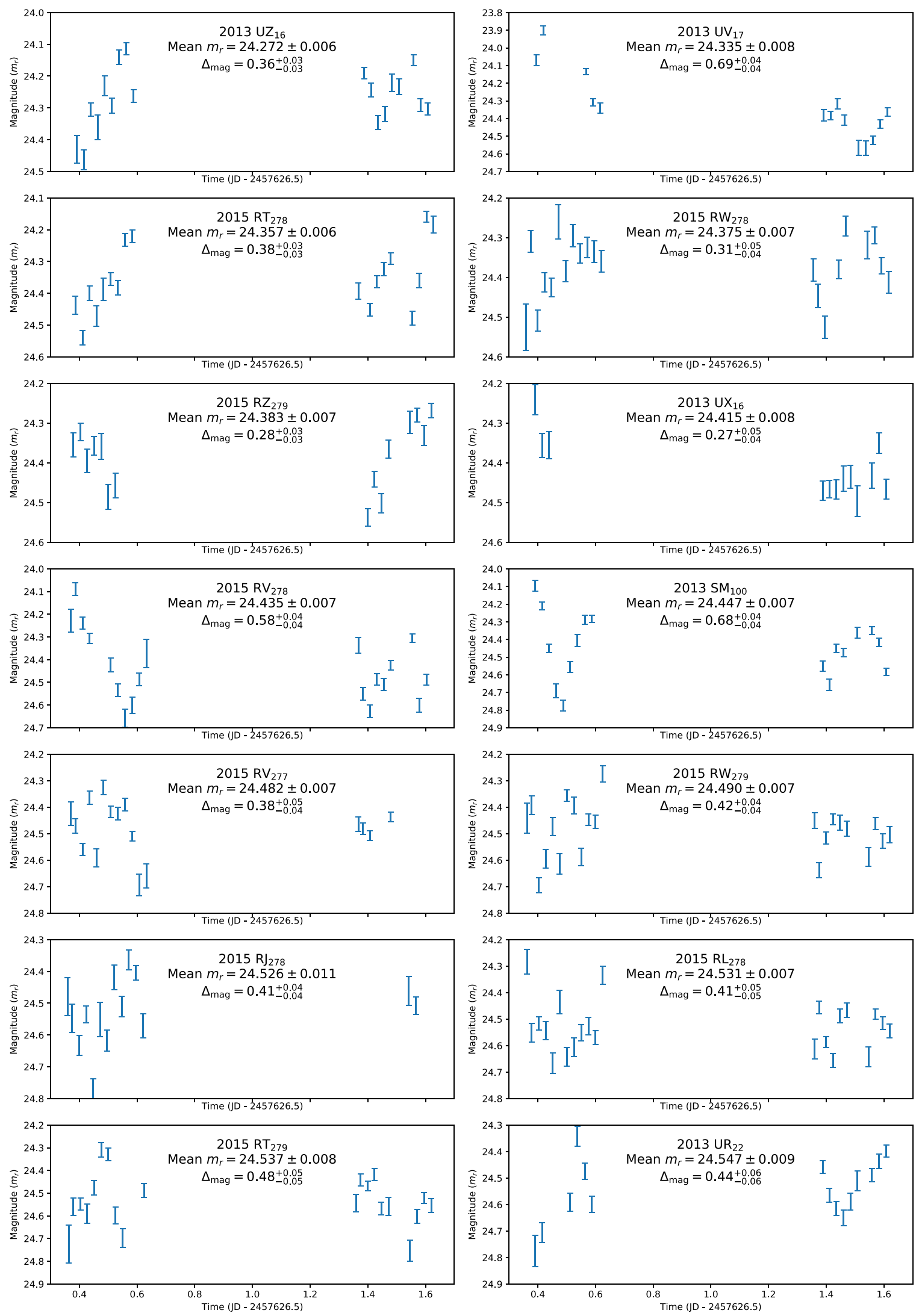

Figure 4. Absolutely calibrated photometry of our TNO sample. Objects $28-41$, ordered by their average magnitude. 

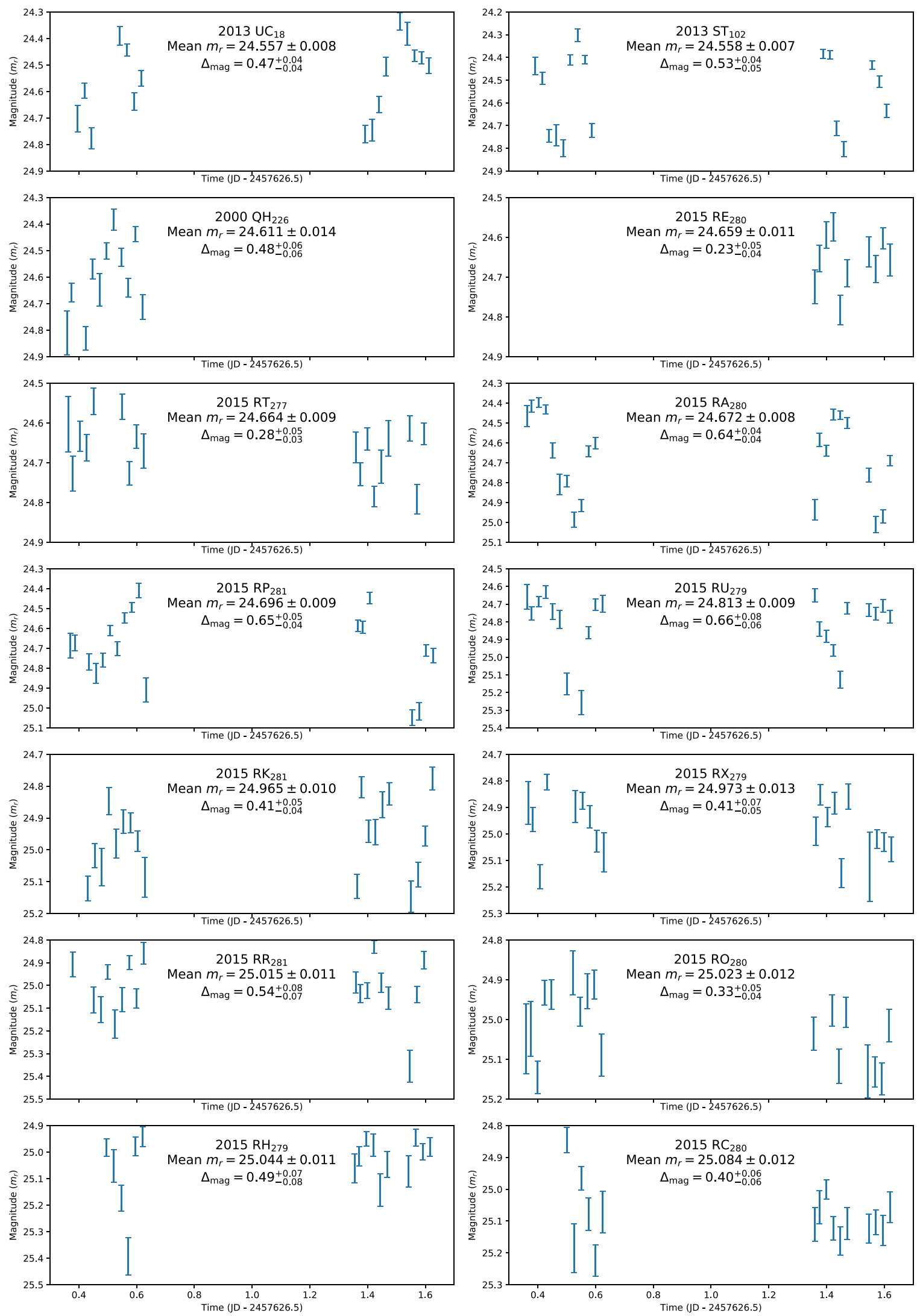

Figure 5. Absolutely calibrated photometry of our TNO sample. Objects $42-55$, ordered by their average magnitude. 

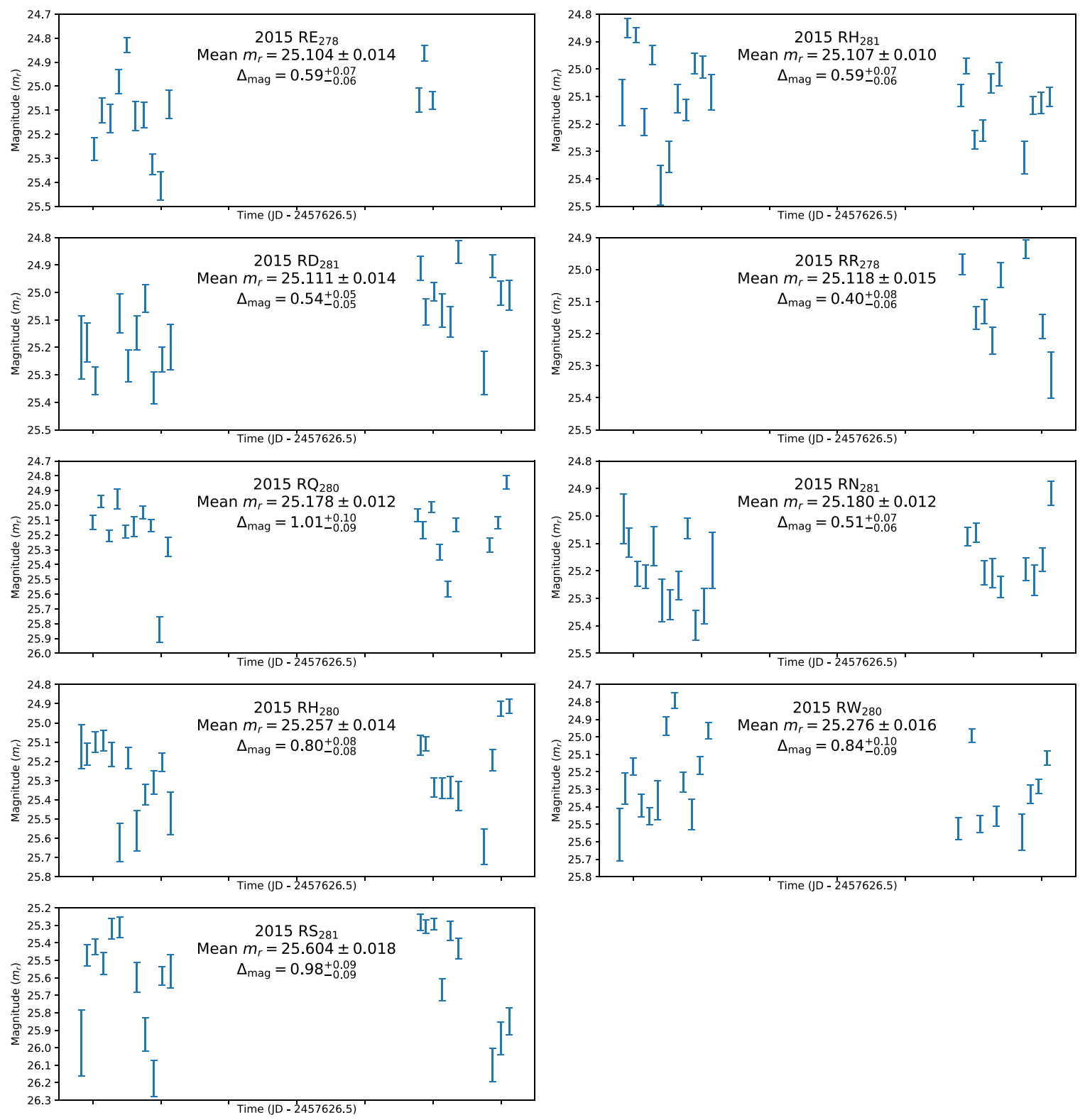

Figure 6. Absolutely calibrated photometry of our TNO sample. Objects 56-64, ordered by their average magnitude.

not in the other. In this work, we use the term "hot population" to refer to all of the dynamically excited populations combined (as done in Fraser et al. 2014, and others); specifically our hot population sample contains everything nonclassical plus classical objects with $i \geqslant 6^{\circ}$. Our "cold classicals" sample consists only of classicals with $i \leqslant 4^{\circ}$. Classicals with $4^{\circ}<$ $i<6^{\circ}$ (two objects in our sample) are excluded from either sample to minimize contamination. The correlation values as well as their significance levels can be found in Table 4 and are simply summarized here. For our samples of 26 cold classical TNOs and 35 hot TNOs, we find that both have similar correlation coefficients to the full sample for the amplitude versus $H_{r}$, but with a lower significance than in the full sample (likely due mostly to the now smaller sample sizes). For the amplitude versus inclination, we find that neither population has a statistically significant correlation, just as in the overall sample. Because the semimajor axis $(a)$ is possibly related to where objects formed, we also tested for correlations between the light-curve variation and $a$ for the full sample and the hot population (the cold population was not tested due to its narrow $a$ range). We find that the semimajor axis is not at all correlated with the variability in the full sample nor in the hot population.

To further test the notion that different populations may have different amplitude distributions, the two-sample AndersonDarling test was used to compare subsamples. The subsamples used were the classical, resonant, and hot and cold populations. We did not look at the scattering and detached objects in this analysis because the sample sizes are too small for any meaningful results. In every case, the subsample was tested against the remainder of the sample (i.e., classicals versus everything nonclassical, etc.). See Table 5 for the resultant $P$ values. No subsample was found to be significantly different from the rest of the sample. 


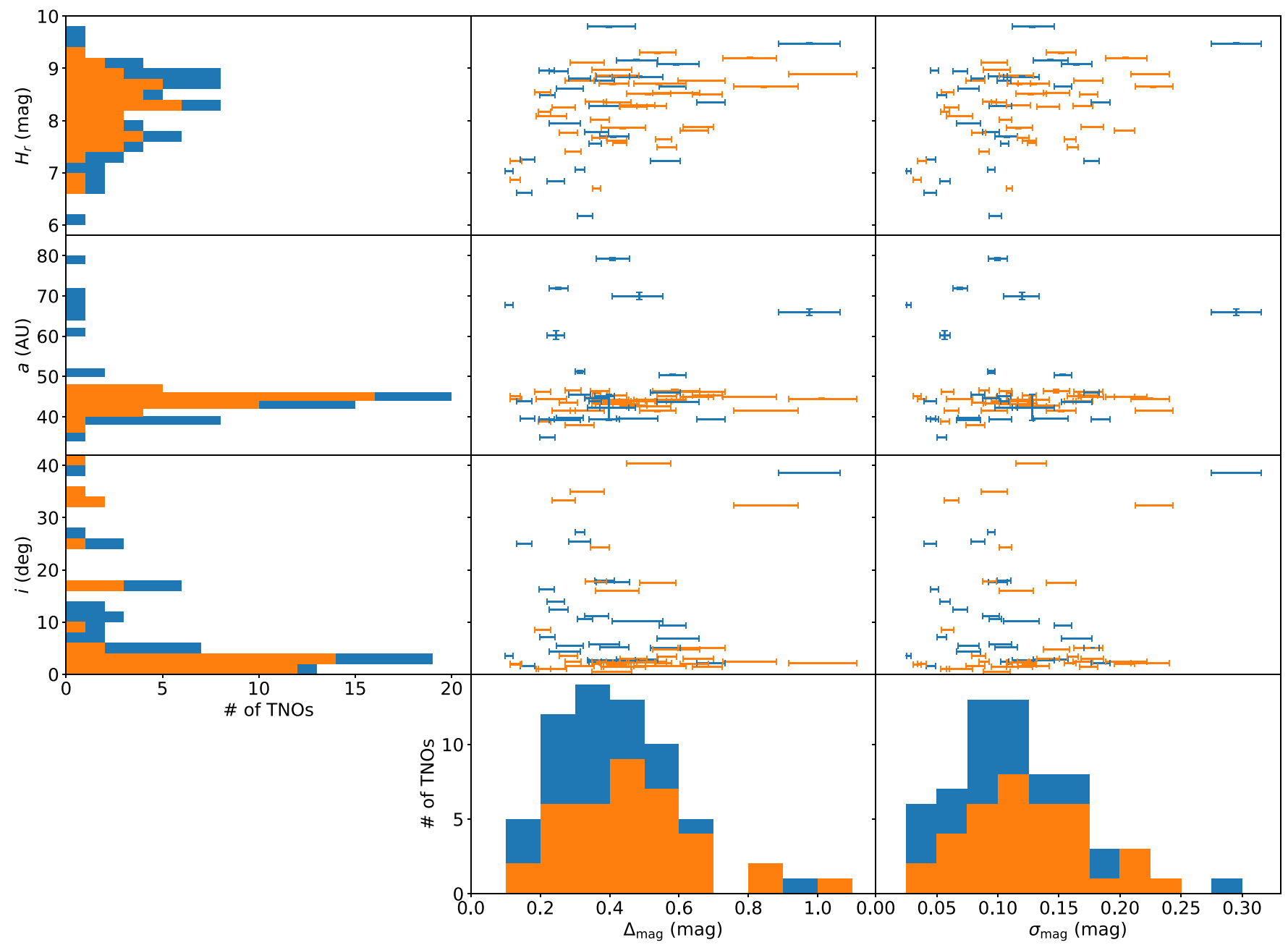

Figure 7. Left and bottom sides: histograms of $H_{r}$ magnitude, semimajor axis $a$, inclination $i, \Delta_{\mathrm{mag}}$, and $\sigma_{\mathrm{mag}}$ of our data. Center and top right: scatter plots of these parameters. Classical TNOs are shown in orange, and nonclassical objects are shown in blue.

Table 4

Spearman Rank Correlation Test Results

\begin{tabular}{lcccc}
\hline \hline Parameter & $\rho_{\Delta_{\text {mag }}}$ & $P_{\Delta_{\text {mag }}}$ & $\rho_{\sigma_{\text {mag }}}$ & $P_{\sigma_{\text {mag }}}$ \\
\hline & & All objects & & \\
$H_{r}$ & $0.37_{-0.03}^{+0.03}$ & 0.003 & $0.31_{-0.02}^{+0.03}$ & 0.013 \\
$a$ & $0.10_{-0.03}^{+0.03}$ & 0.43 & $0.11_{-0.02}^{+0.02}$ & 0.41 \\
$i$ & $-0.05_{-0.03}^{+0.03}$ & 0.7 & $-0.07_{-0.02}^{+0.02}$ & 0.60 \\
& & Cold classicals & & \\
$H_{r}$ & $0.43_{-0.05}^{+0.04}$ & 0.029 & $0.30_{-0.04}^{+0.03}$ & 0.14 \\
$i$ & $0.25_{-0.05}^{+0.05}$ & 0.21 & $0.38_{-0.04}^{+0.03}$ & 0.06 \\
& & Hot populations & & \\
$H_{r}$ & $0.42_{-0.05}^{+0.04}$ & 0.013 & $0.41_{-0.04}^{+0.03}$ & 0.015 \\
$a$ & $0.04_{-0.05}^{+0.05}$ & 0.79 & $0.04_{-0.04}^{+0.04}$ & 0.79 \\
$i$ & $0.08_{-0.04}^{+0.05}$ & 0.6 & $0.03_{-0.03}^{+0.03}$ & 0.85 \\
\hline
\end{tabular}

Note. Tests for correlation between the measured light-curve variation $\Delta_{\text {mag }}$ and $\sigma_{\mathrm{mag}}$ and the parameter listed in the first column.

\subsection{Period Analysis}

The rotation periods of TNOs range from $3.9 \mathrm{hr}$ (Haumea) to $154 \mathrm{hr}$ (Pluto), with a mean of 7-9 hr (Duffard et al. 2009; Benecchi \& Sheppard 2013; Thirouin et al. 2016); there are clearly a number of assumptions and biases that go into this mean,
Table 5

Two-sample Anderson-Darling Test Results

\begin{tabular}{lccr}
\hline \hline Subpopulation & $N_{\text {obj }}$ & $P_{\Delta_{\text {mag }}}$ & $P_{\sigma_{\text {mag }}}$ \\
\hline Classicals & 37 & $0.15_{-0.07}^{+0.10}$ & $0.11_{-0.05}^{+0.06}$ \\
Resonant & 15 & $0.8 \pm 0.2$ & $0.76_{-0.14}^{+0.12}$ \\
Hot & 35 & $0.13_{-0.06}^{+0.09}$ & $0.09_{-0.03}^{+0.06}$ \\
Cold & 26 & $0.4 \pm 0.2$ & $0.29_{-0.09}^{+0.12}$ \\
\hline
\end{tabular}

Note. Tests for whether two samples are likely to have been drawn from the same source population. Here we test whether the light-curve variability (measured by $\Delta_{\text {mag }}$ and $\sigma_{\text {mag }}$ ) distribution is different for different dynamical subpopulations. In every case here, a subsample is tested against the remainder of our sample (e.g., the resonant objects against everything that is not resonant).

not the least of which is the aliasing effects from observations on consecutive nights and the fact that tracking low-amplitude and slowly varying objects requires significant telescope time. Both of these biases exist in our data set; nevertheless, we seek to use the data we present here to at least place limits on the rotation periods and amplitudes of the objects in our sample. In the few cases where our data allow for more extensive interpretation, we proceed as appropriate for each object. We attempt to fit periods to our light-curve data using two methods: a phase dispersion minimization (PDM) method and a power spectral density (PSD) 
Table 6

Best Periods from Two Methods, in Hours

\begin{tabular}{lcccc}
\hline \hline Object & Period & & & \\
PdM & Period & \\
PDS & (hour) & \\
\hline $2013 \mathrm{UL}_{15}$ & $11.76 \pm 0.02$ & $(41.3 \%)$ & $11.80 \pm 0.03$ & $(100.0 \%)$ \\
$2013 \mathrm{UC}_{18}$ & $15.66 \pm 0.23$ & $(80.7 \%)$ & $15.70 \pm 0.08$ & $(68.8 \%)$ \\
$2013 \mathrm{UP}_{15}$ & $16.02 \pm 0.09$ & $(37.7 \%)$ & $16.00 \pm 0.07$ & $(100.0 \%)$ \\
$2013 \mathrm{UM}_{15}$ & $6.44 \pm 0.03$ & $(81.0 \%)$ & $6.40 \pm 0.04$ & $(49.0 \%)$ \\
$2013 \mathrm{UN}_{15}$ & $20.30 \pm 0.08$ & $(79.2 \%)$ & $20.30 \pm 0.09$ & $(43.6 \%)$ \\
$2015 \mathrm{RU}_{277}$ & $6.58 \pm 0.05$ & $(84.4 \%)$ & $6.50 \pm 0.03$ & $(99.3 \%)$ \\
$2015 \mathrm{RG}_{281}$ & $6.03 \pm 0.02$ & $(98.2 \%)$ & $6.07 \pm 0.04$ & $(54.3 \%)$ \\
$2015 \mathrm{RV}_{278}$ & $13.54 \pm 0.04$ & $(94.8 \%)$ & $13.70 \pm 0.14$ & $(74.9 \%)$ \\
$2015 \mathrm{RU}_{279}$ & $14.38 \pm 0.08$ & $(74.0 \%)$ & $14.40 \pm 0.09$ & $(41.3 \%)$ \\
$2015 \mathrm{RT}_{279}$ & $11.34 \pm 0.11$ & $(30.3 \%)$ & $11.30 \pm 0.09$ & $(71.9 \%)$ \\
$2003 \mathrm{SP}_{317}$ & $12.45 \pm 0.04$ & $(100.0 \%)$ & $12.45 \pm 0.04$ & $(100.0 \%)$ \\
$2015 \mathrm{RA}_{280}$ & $12.66 \pm 0.05$ & $(99.7 \%)$ & $12.72 \pm 0.04$ & $(100.0 \%)$ \\
\hline
\end{tabular}

Note. Numbers in parentheses are the percentage of resampled light curves that had a "best" period within $5 \sigma$ of the reported value. Only objects where the two methods agree on the best period are included. As these objects are small, their variability is most likely shape-dominated; shape dominated light curves have two peaks per rotation of the object. These periods have thus been doubled to represent the rotation period of the object.

(This table is available in machine-readable form.)

method. Because our TNOs are all small enough that we do not expect them to be spherical, they should all have double-peaked periods. The two light-curve peaks of an elongated TNO are often difficult to distinguish (for a perfectly symmetrical ellipsoid, the two peaks would be identical). These methods, therefore, simply identify the period of the light curve, not the TNO. We double the single-peaked periods found by our two period methods to get the most likely rotation period of the TNO.

Because our observing window is about $30 \mathrm{hr}$ (from the start of night one to the end of night two), we are able to sample rotation periods from the rotational break-up-limit of $3.3 \mathrm{hr}$ (Romanishin \& Tegler 1999) to about $60 \mathrm{hr}$ (where we sample half a rotation, which is a full peak-to-peak cycle for a double-peaked light curve). For the PSD method, we, therefore, fold the light curves using every period from 1.0 to $30.0 \mathrm{hr}$ in $0.1 \mathrm{hr}$ intervals. The Fourier coefficients are calculated from each folded light curve; the sum of the squares of the Fourier coefficients as a function of the period is the PSD. The period that has the highest PSD value is selected as the most likely period of the TNO. In the PDM method, we fit the data points for each object using a modified PDM (Stellingwerf 1978; Buie et al. 2018). This method goes through every possible period, folds the data, and fits a secondorder Fourier series to each folded light curve. The quality of each fit is captured from the residuals; the fits are ranked and the fit with the highest quality is selected.

Table 6 lists the periods found from these two methods (converted into double-peak periods) for objects where the two methods agree; Figure 8 shows the light curves folded at those periods. When the two methods do not agree, it is likely evidence that we do not have enough data to determine the period with confidence. The PDM curves from our period analysis are available in the extended Figure 10. Although the mean period from our data set analysis is somewhat greater than that given in the literature ( $12.3 \mathrm{hr}$ compared with 7-9 hr; Duffard et al. 2009; Thirouin et al. 2010; Benecchi \& Sheppard 2013), it is important to recognize that the periods we are reporting are based on single epoch measurements without the same fidelity of many of the light curves in the literature. The literature contains both single- and double-peaked light curves and light curves of binary objects. We cannot distinguish among these interpretations and have assumed a double-peaked light curve for all objects, so one should not overinterpret this apparent result from our data set. This sample of 12 periods is insufficient to draw any conclusions, other than the fact that we would need a larger baseline than two consecutive $6 \mathrm{hr}$ nights to determine the period of a larger fraction of objects. Adding a third night and observing closer to opposition (when the targets are visible for $8 \mathrm{hr}$ ) would allow determination of periods for a much larger fraction of light curves. We, therefore, leave discussions of the period distribution for future work.

\section{Discussion and Conclusions}

\subsection{Implications for Large Surveys}

Large variability can have an influence on whether or not an object is discovered in a magnitude-limited survey. Most large surveys use an automated moving object pipeline to find TNO candidates. For the Canada-France Ecliptic Plane Survey (Kavelaars et al. 2009; Petit et al. 2011), OSSOS, and the Alexandersen et al. (2016) survey, TNOs were identified using three discovery images with one hour between each. Nonstationary sources within three images of a field are linked together as a candidate TNO detection; various constraints on the rate, angle, and linearity of motion are imposed, as well as a requirement of similar magnitudes, in order to limit the number of false candidates. If a TNO's magnitude is highly variable, this might leave it undetected, either because it might be too faint to be detected in one of the three images or, if the constraints in the detection pipeline are too tight, because the pipeline might not consider the three points to be the same object because it has different magnitudes.

In our sample of $63 \mathrm{TNO}$ light curves (most with observations on both nights), we find that only $6 \pm 1$ have a $\Delta_{\text {mag }}<0.2 \mathrm{mag}$, while $31 \pm 2$ (just under half the sample) have a $\Delta_{\mathrm{mag}}>$ $0.4 \mathrm{mag}$, and $11 \pm 1$ have $\Delta_{\mathrm{mag}}>0.6 \mathrm{mag} .{ }^{17}$ However, those variations are usually over many hours. In order to evaluate the impact that light-curve variation could have on shorter timescales, we measured the typical variation on 1-2 hr timescales. Since our images for these light curves are typically spaced 36 minutes apart, we calculated the $\Delta_{\text {mag }}$ for all $1.25 \mathrm{hr}$ (3 images), $1.85 \mathrm{hr}$ (4 images) and $2.45 \mathrm{hr}$ (5 images) subsets of our light curves. We find that in $1.25 \mathrm{hr}$, the median value of the $\Delta_{\text {mag }}$ is $0.13 \mathrm{mag}$ and the standard deviation of the $\Delta_{\text {mag }}$ distribution is $0.12 \mathrm{mag}$. For $1.85 \mathrm{hr}$, the median and standard deviation are 0.16 and 0.13 , respectively, while for $2.45 \mathrm{hr}$ they are 0.19 and $0.14 \mathrm{mag}$, respectively. Figure 9 shows the $\Delta_{\text {mag }}$ distribution within these three time spans. The moving object pipeline used for the Alexandersen et al. (2016) survey and for OSSOS only had the weak constraint that the faintest and brightest points within the three discovery images (spaced about $1 \mathrm{hr}$ apart) had to have a flux ratio of less than 4 , which corresponds to a magnitude difference of about 1.5 mag. In our data from this work, we never see variability greater than $1.2 \mathrm{mag}$ within a $2 \mathrm{hr}$ window; therefore, it seems highly unlikely that a real objects was rejected by the detection pipeline due to natural variability. Because the overall average variability seen within our data is much larger than the average variability measured within $1.25-2.45 \mathrm{hr}$, this demonstrates the

\footnotetext{
17 The uncertainties quoted in this sentence on the number of objects come from resampling the light-curve photometry 1000 times and calculating the values for each resampled set.
} 

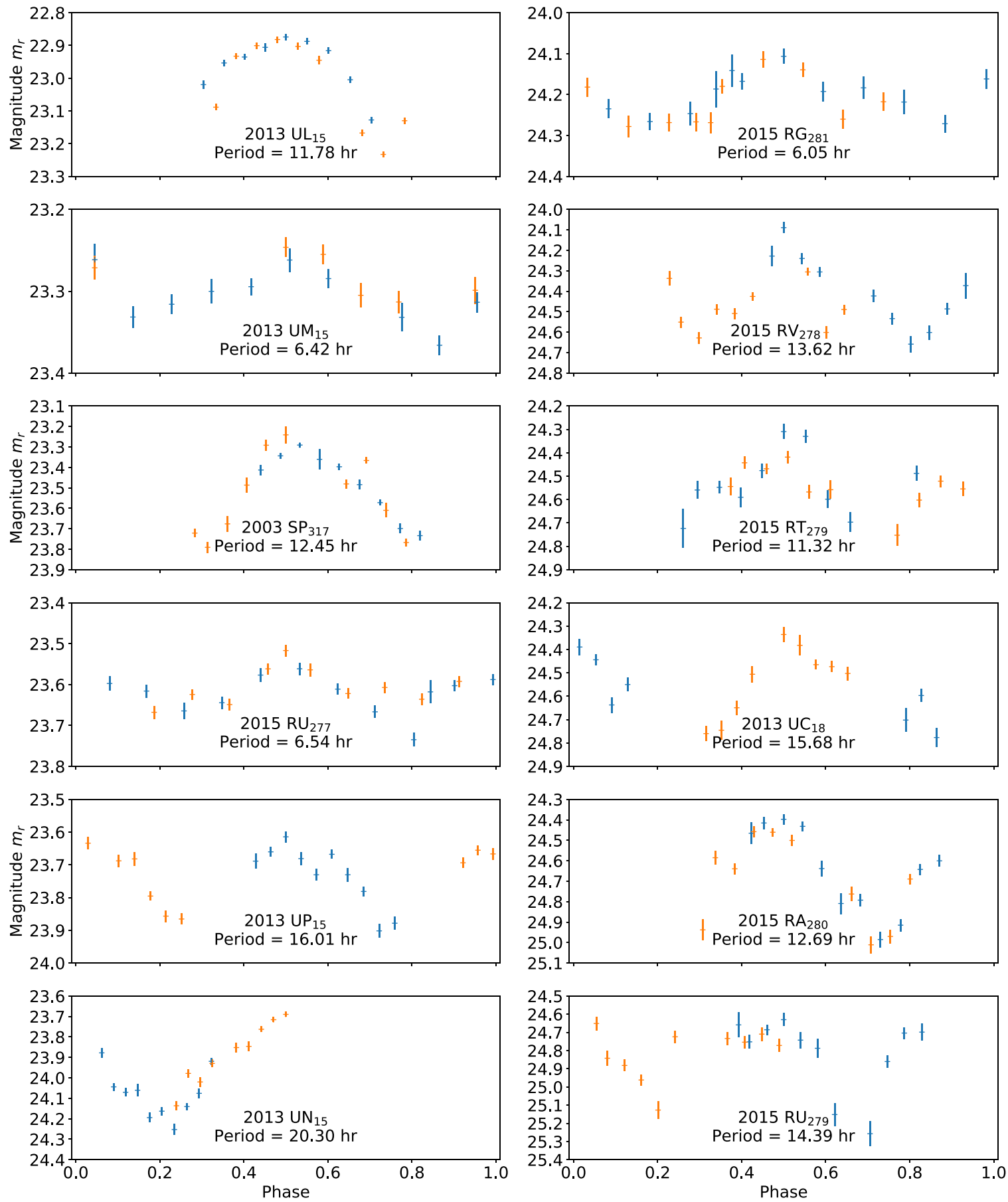

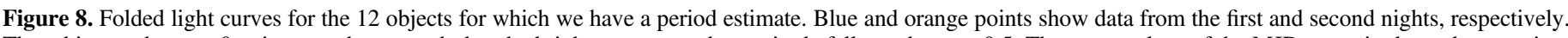

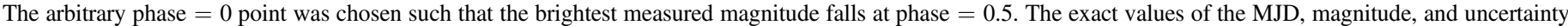
of all observations can be found in Table 3 .

perhaps obvious point that the average magnitude measured within a few hours is not an accurate measurement of the true average magnitude of the TNO. Discovery images from surveys like CFEPS, OSSOS, and the Alexandersen et al. (2016) survey all depend on measuring the magnitude in three discovery images; the fact that these measured magnitudes are potentially several tenths of a magnitude from the true average magnitude should be included when performing survey simulations and model analyses.

Variability is important to consider when measuring photometric colors of TNOs as well. Because most facilities do not have the ability to observe in multiple bandpasses simultaneously, the images in different filters must be taken in sequence. If the object is faint and thus requires a long exposure time in some/each filter, its variability could cause an erroneous measurement of the color if the brightness varies significantly within the timespan of the observations. For accurate photometric colors, it is, therefore, important to account for light-curve variability, except in cases of very short (thus, nearly simultaneous) exposure sequences for bright objects. This can be done by repeating observations of the filter that requires least exposure time several times during a sequence (for example, $r-g-r-J-r$ ), as has become common 


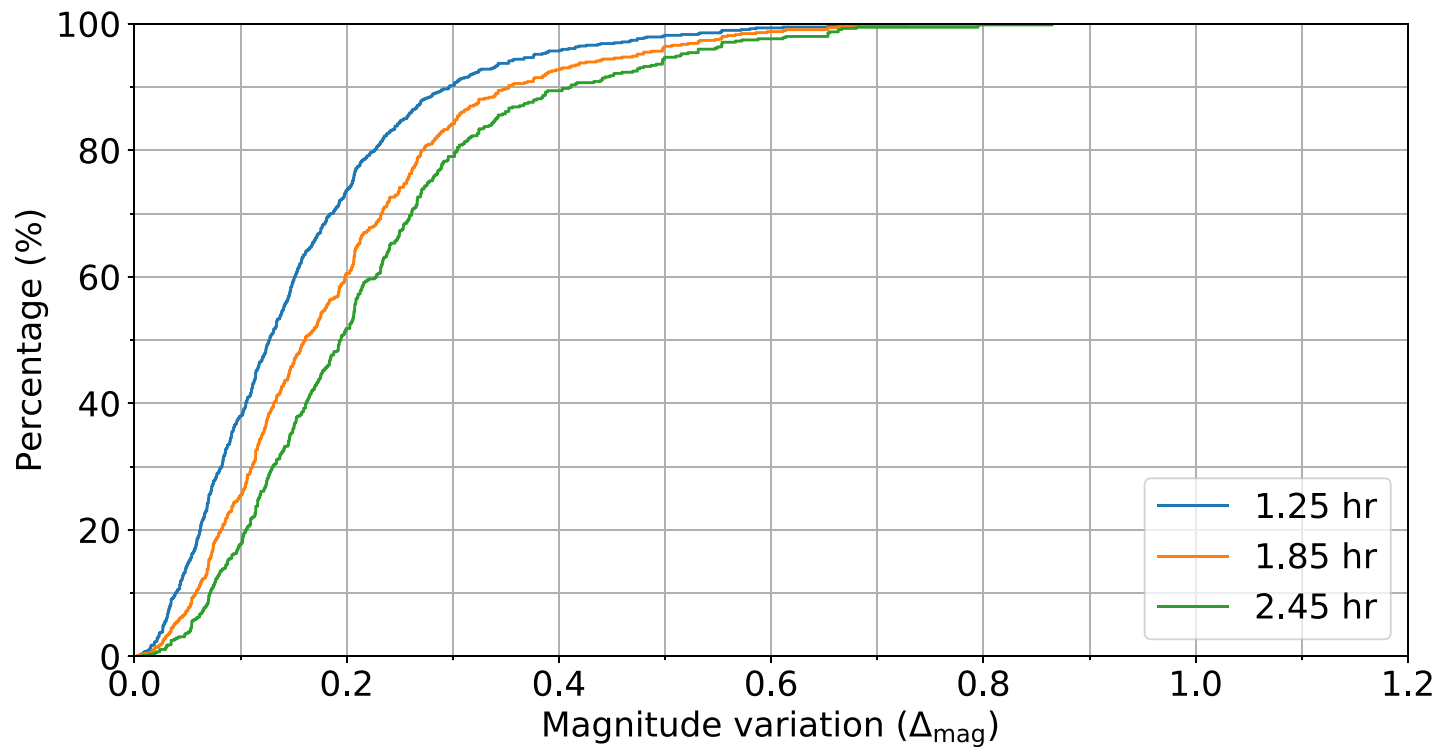

Figure 9. Cumulative distribution of the magnitude variation (the maximum minus the minimum: $\Delta_{\text {mag }}$ ) within $1.25 \mathrm{hr}$ (blue, top), $1.85 \mathrm{hr}$ (orange, middle), and $2.45 \mathrm{hr}$ (green, bottom) sections of light curves.

practice, to get a rough idea of the light-curve variation during the sequence. Alternatively, if some of the filters require enough exposure time to warrant multiple exposures, those exposures should be spread out in the sequence (for example, the $r-g-J-g-r$ sequence used by Bannister et al. 2017). Of course, this relies on having fast filter changes and can, therefore, not be done with HSC, which has a filter exchange time of about 30 minutes. It should be obvious that photometry acquired on different days cannot be used to calculate reliable colors unless the light curve is known, so TNO colors reported from surveys like the HSC Subaru Strategic Program (HSC SSP; Aihara et al. 2018; Terai et al. 2018) or the LSST survey should be seen as very rough color estimates with a likely uncertainty of $\sim 0.2$ mag.

Expected to start science operations in 2022, LSST will provide an inventory of the trans-Neptunian region down to a $5 \sigma$ limiting magnitude of approximately 24.6 in $r$ and 24.8 in $g$ (LSST Science Collaboration et al. 2009; Ivezic et al. 2019). LSST is expected to discover over 40,000 TNOs during its planned 10 years of science operations (LSST Science Collaboration et al. 2009). During this period, TNOs within the survey footprint will be imaged hundreds of times by LSST in multiple filters (ugrizy), producing sparsely sampled light curves. Our HSC variability study overlaps the size and magnitude range that LSST will be sensitive to. Thus, the variability we observe can be taken as a minimum value for the variability LSST will see for its TNO sample. Over a few hr baseline, $90 \%$ of our HSC sample vary in brightness up to $0.4 \mathrm{mag}$. This is more variability than shallower past wide-field solar system surveys had to deal with. As detailed above, OSSOS, which our sample is derived from, had no significant brightness/variability constraints when identifying moving objects (Bannister et al. 2018). This may be important in the development of the LSST moving object detection pipeline and for consideration when assessing the TNO detection completeness for LSST. Additionally, in the sparse light curves LSST will produce, objects with more than 0.5 mag variation can potentially be flagged as extreme shapes in need of further highercadence follow-up observations.

Finally, we note that objects in this size range do not have enough mass to ensure hydrostatic equilibrium, so the variability seen is, therefore, most likely caused by the objects being highly nonspherical. Given the correlation between the absolute $H_{r}$ magnitude and variability shown above, it is unreasonable to expect that even smaller object can be modeled as roughly spherical shapes. A number of surveys have searched for serendipitous stellar occultations by small (diameters $\sim 1 \mathrm{~km}$, roughly corresponding to $H_{r} \sim 16-19$ depending on albedo) TNOs in order to measure the size distribution of such objects (Roques et al. 2003; Bickerton et al. 2008; Liu et al. 2008; Bianco et al. 2009; Schlichting et al. 2012; Zhang et al. 2013; Lehner et al. 2016). TNOs in this size range are in the Fresnel regime when measuring from the ground at visible wavelengths, and their occultation shadows thus exhibit significant diffraction features (Roques et al. 1987; Nihei et al. 2007). Most survey teams use the approximation of spherical occulting objects in order to simplify the calculation of the occultation event parameters, but when nonspherical shapes are considered, there may be significant effects on the resulting occultation shadows (Roques et al. 1987; Castro-Chacón 2019). Obviously, we did not measure light curves of such small objects in this survey; however, based on our observations of larger $\left(8>H_{r}>10\right)$ TNOs, we propose that these surveys need to consider the expectation of nonspherical objects when defining event detection criteria, characterizing any occultation events, or estimating any survey detection efficiencies.

\subsection{Contact Binaries}

To date, in the trans-Neptunian belt, only $2001 \mathrm{QG}_{298}$ has been confirmed as a contact binary (Sheppard \& Jewitt 2004; Lacerda et al. 2014) besides the New Horizons flyby target $2014 \mathrm{MU}_{69}$. This confirmation is based on the large light-curve amplitude of $\Delta_{\text {mag }} \geqslant 0.9 \mathrm{mag}$, and the $\mathrm{U} / \mathrm{V}$ shape at the minimum/maximum of the brightness. However, such a large amplitude is only observed if the two components are seen equator-on (or almost equator-on). If the two components are not aligned with the viewer's line of sight, the amplitude will be smaller (simple geometry; Lacerda 2011; Lacerda et al. 2014; Thirouin et al. 2017; Thirouin \& Sheppard 2017, 2018).

Our sparse light curves reveal no obvious contact binaries, although they do contain candidates that should be observed further. None of the light curves reported here present a definite 
full peak-to-peak amplitude larger than 0.9 mag. $2015 \mathrm{RQ}_{280}$, $2015 \mathrm{RS}_{281}$, and $2015 \mathrm{RW}_{280}$ have a $\Delta_{\text {mag }}$ around $1 \mathrm{mag}$, but the light curves are too noisy for drawing conclusions. Several objects like $2003 \mathrm{SP}_{317}, 2015 \mathrm{RB}_{280}, 2013 \mathrm{UN}_{15}$, and 2013 $\mathrm{SM}_{100}$ display a $\Delta_{\text {mag }}>0.5 \mathrm{mag}$. Unfortunately, because we do not have a full maximum and minimum showing a clear $\mathrm{U}$ and $\mathrm{V}$ shape, it is difficult to classify them as likely contact binaries. More observations are required in order to confirm whether these objects are contact binaries or simply highly elongated bodies.

\subsection{Conclusions}

We obtained a large sample of sparse partial light curves of 65 TNOs, most of which are small $\left(H_{r}>7\right)$ and, therefore, fainter than have typically been studied before, with up to 22 observations in acceptable conditions over two nights. While the variability for many of these objects might only be a lower limit because we have not observed the full rotation of the object, we can draw some tentative conclusions. As seen by Benecchi \& Sheppard (2013), we confirm that the amplitude of the light curve correlates with the absolute magnitude. However, in contrast, we do not see a statistically significant correlation with the inclination.

We find that a large fraction of our observed TNOs have large brightness variations, even on $1-2 \mathrm{hr}$ timescales. Thirty one objects (just under half the sample) have a $\Delta_{\text {mag }}>0.4$ mag over the course of our observations, while the median variability within a $1.85 \mathrm{hr}$ timespan was $0.16 \mathrm{mag}$. TNO variability must be accounted for when simulating/modeling discovery surveys like OSSOS and LSST, as well as when observing nonsimultaneous TNO colors.

In this pilot study, we did not find any significant difference in amplitude distribution between different dynamical classes. However, our sample included very few objects in the scattering and detached classes. Future work should, therefore, target more of these objects in order to have similar sample sizes for each population. We were able to estimate periods for 12 of the 65 TNOs. However, these periods are not certain due to aliasing. In order to estimate periods for a larger fraction of the sample and reduce aliasing effects, a longer baseline of observations is needed.

This paper is based on data collected at the Subaru Telescope and retrieved from the HSC data archive system, which is operated by Subaru Telescope and Astronomy Data Center at National Astronomical Observatory of Japan. The access to Subaru telescope from Taiwan is supported by the Academia Sinica Institute of Astronomy and Astrophysics. The authors wish to recognize and acknowledge the very significant cultural role and reverence that the summit of Maunakea has always had within the indigenous Hawaiian community. We are most fortunate to have the opportunity to conduct observations from this mountain. We would also like to acknowledge the maintenance, cleaning, administrative, and support staff at academic and telescope facilities, whose labor maintains the spaces where astrophysical inquiry can flourish.

This work is based on TNO discoveries obtained with MegaPrime/MegaCam, a joint project of the Canada-FranceHawaii Telescope (CFHT) and CEA/DAPNIA, at CFHT, which is operated by the National Research Council (NRC) of Canada, the Institute National des Sciences de l'Universe of the Centre National de la Recherche Scientifique (CNRS) of France, and the University of Hawaii. A portion of the access to the CFHT was made possible by the Academia Sinica
Institute of Astronomy and Astrophysics, Taiwan. This research used the facilities of the Canadian Astronomy Data Centre operated by the National Research Council of Canada with the support of the Canadian Space Agency.

M.E.S. was supported by the Gemini Observatory, which is operated by the Association of Universities for Research in Astronomy, Inc., on behalf of the international Gemini partnership of Argentina, Brazil, Canada, Chile, and USA. M.B. acknowledges support from UK STFC grant ST/L000709/1. K.V. acknowledges support from NASA grants NNX14AG93G and NNX15AH59G. S.D.B. acknowledges support by the NASA under Grant/Contract/Agreement No. NNX15AE04G issued through the SSO Planetary Astronomy Program. M.R.E. acknowledges support from the ASIAA summer student program.

This paper makes use of software developed for the Large Synoptic Survey Telescope. We thank the LSST Project for making their code available as free software at http://dm.lsst.org. This research has made use of NASA's Astrophysics Data System Bibliographic Services. This research made use of SciPy (Jones et al. 2001), NumPy (van der Walt et al. 2011), matplotlib (a Python library for publication quality graphics Hunter 2007) and Astropy (a community-developed core Python package for Astronomy Astropy Collaboration et al. 2013; The Astropy Collaboration et al. 2018). This research made use of ds9, a tool for data visualization supported by the Chandra X-ray Science Center (CXC) and the High Energy Astrophysics Science Archive Center (HEASARC) with support from the James Webb Space Telescope Mission office at the Space Telescope Science Institute for 3D visualization. IRAF is distributed by the National Optical Astronomy Observatory, which is operated by the Association of Universities for Research in Astronomy (AURA) under cooperative agreement with the National Science Foundation (Tody 1993).

The Pan-STARRS1 Surveys (PS1) and the PS1 public science archive have been made possible through contributions by the Institute for Astronomy, the University of Hawaii, the PanSTARRS Project Office, the Max Planck Society and its participating institutes, the Max Planck Institute for Astronomy Heidelberg, and the Max Planck Institute for Extraterrestrial Physics Garching, The Johns Hopkins University, Durham University, the University of Edinburgh, the Queen's University Belfast, the Harvard-Smithsonian Center for Astrophysics, the Las Cumbres Observatory Global Telescope Network Incorporated, the National Central University of Taiwan, the Space Telescope Science Institute, NASA under grant No. NNX08AR22G issued through the Planetary Science Division of the NASA Science Mission Directorate, the National Science Foundation grant No. AST-1238877, the University of Maryland, Eotvos Lorand University (ELTE), the Los Alamos National Laboratory, and the Gordon and Betty Moore Foundation.

The acknowledgments were compiled in part using the Astronomy Acknowledgment Generator.

Facilities: Subaru (HSC), PS1.

Software: Python (van Rossum \& de Boer 1991), Matplotlib (Hunter 2007), NumPy (van der Walt et al. 2011), SciPy (Jones et al. 2001), TRIPPy (Fraser et al. 2016), Ureka (Hirst et al. 2014), IRAF (Tody 1993), Astropy (Astropy Collaboration et al. 2013; The Astropy Collaboration et al. 2018), SExtractor (Bertin \& Arnouts 1996), ds9 Joye \& Mandel (2003).

\section{Appendix}

The PDM curves from our period analysis are available in the extended Figure 10. 

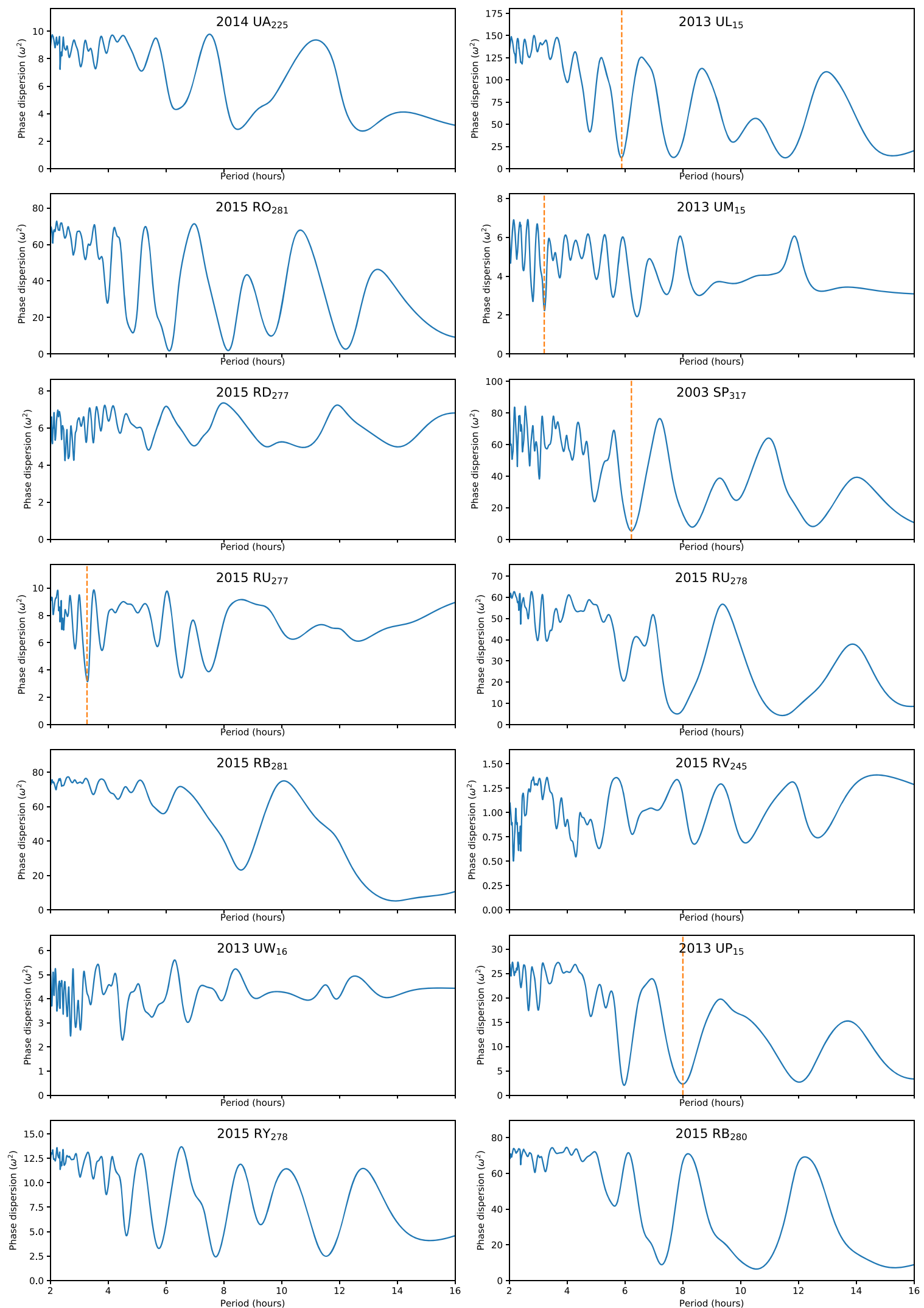

Figure 10. PDM plot from our period analysis. Vertical dashed orange lines show our favored single-peak period for the objects listed in Table 6 and shown in Figure 8. Objects $0-13$, ordered by average magnitude shown here. (An extended version of this figure is available.) 


\section{ORCID iDs}

Mike Alexandersen (iD https://orcid.org/0000-0003-4143-8589

Susan D. Benecchi (i) https://orcid.org/0000-0001-8821-5927

Ying-Tung Chen (英同陳) (1) https:// orcid.org/0000-00017244-6069

Marielle R. Eduardo (i) https://orcid.org/0000-0002-

0760-1584

Audrey Thirouin (iD https://orcid.org/0000-0002-1506-4248

Megan E. Schwamb (iD https://orcid.org/0000-0003-

4365-1455

Matthew J. Lehner (10 https://orcid.org/0000-0003-4077-0985

Shiang-Yu Wang (王祥宇) (iD https://orcid.org/0000-0001-

6491-1901

Michele T. Bannister (iD https://orcid.org/0000-0003-

3257-4490

JJ. Kavelaars (iD https://orcid.org/0000-0001-7032-5255

Jean-Marc Petit (iD https://orcid.org/0000-0003-0407-2266

Kathryn Volk (DD https://orcid.org/0000-0001-8736-236X

\section{References}

Aihara, H., Arimoto, N., Armstrong, R., et al. 2018, PASJ, 70, S4

Alexandersen, M., Gladman, B., Kavelaars, J. J., et al. 2016, AJ, 152, 111

Astropy Collaboration, Robitaille, T. P., Tollerud, E. J., et al. 2013, A\&A, 558, A33

Bannister, M. T., Gladman, B. J., Kavelaars, J. J., et al. 2018, ApJS, 236, 18

Bannister, M. T., Kavelaars, J. J., Petit, J.-M., et al. 2016, AJ, 152, 70

Bannister, M. T., Schwamb, M. E., Fraser, W. C., et al. 2017, ApJL, 851, L38

Batygin, K., Brown, M. E., \& Fraser, W. C. 2011, ApJ, 738, 13

Benecchi, S. D., \& Sheppard, S. S. 2013, AJ, 145, 124

Bertin, E., \& Arnouts, S. 1996, A\&AS, 117, 393

Bianco, F. B., Protopapas, P., MacLeod, B. A., et al. 2009, AJ, 138, 568

Bickerton, S. J., Kavelaars, J. J., \& Welch, D. L. 2008, AJ, 135, 1039

Bosch, J., Armstrong, R., Bickerton, S., et al. 2018, PASJ, 70, S5

Buie, M. W., Zangari, A. M., Marchi, S., Levison, H. F., \& Mottola, S. 2018, AJ, 155, 245

Castro-Chacón, J. H., Reyes-Ruiz, M., Lehner, M. J., et al. 2019, PASP, 131, 064401

Duffard, R., Ortiz, J. L., Thirouin, A., Santos-Sanz, P., \& Morales, N. 2009, A\&A, 505, 1283

Fraser, W., Alexandersen, M., Schwamb, M. E., et al. 2016, AJ, 151, 158

Fraser, W. C., \& Brown, M. E. 2012, ApJ, 749, 33

Fraser, W. C., Brown, M. E., Morbidelli, A., Parker, A., \& Batygin, K. 2014, ApJ, 782, 100

Gladman, B., Marsden, B. G., \& Vanlaerhoven, C. 2008, in The Solar System Beyond Neptune, ed. M. A. Barucci et al. (Tucson, AZ: Univ. Arizona Press), 43

Hahn, J. M., \& Malhotra, R. 2005, AJ, 130, 2392

Hirst, P., Slocum, C., Turner, J., et al. 2014, in ASP Conf. Ser. 485, Astronomical Data Analysis Software and Systems XXIII, ed. N. Manset \& P. Forshay (San Francisco, CA: ASP), 335

Hunter, J. D. 2007, CSE, 9, 90

Ivezic, Z., Kahn, S. M., Tyson, J. A., et al. 2019, ApJ, 873, 111

Jones, E., Oliphant, T., Peterson, P., et al. 2001, SciPy: Open Source Scientific Tools for Python v.0.17.1, http://www.scipy.org/

Joye, W. A., \& Mandel, E. 2003, in ASP Conf. Ser. 295, Astronomical Data Analysis Software and Systems XII, ed. H. E. Payne, R. I. Jedrzejewski, \& R. N. Hook (San Francisco, CA: ASP), 489
Kaula, W. M., \& Newman, W. I. 1992, AdSpR, 12, 3

Kavelaars, J. J., Jones, R. L., Gladman, B. J., et al. 2009, AJ, 137, 4917

Kortenkamp, S. J., Malhotra, R., \& Michtchenko, T. 2004, Icar, 167, 347

Lacerda, P. 2011, AJ, 142, 90

Lacerda, P., McNeill, A., \& Peixinho, N. 2014, MNRAS, 437, 3824

Lawler, S. M., Shankman, C., Kavelaars, J. J., et al. 2018, AJ, 155, 197

Lehner, M. J., Wang, S.-Y., Reyes-Ruiz, M., et al. 2016, Proc. SPIE, 9906, $99065 \mathrm{M}$

Levison, H. F., Morbidelli, A., Van Laerhoven, C., Gomes, R., \& Tsiganis, K. 2008, Icar, 196, 258

Li, J., Zhou, L.-Y., \& Sun, Y.-S. 2008, ChA\&A, 32, 409

Liu, C.-Y., Chang, H.-K., Liang, J.-S., \& King, S.-K. 2008, MNRAS, 388, L44

LSST Science Collaboration, Abell, P. A., Allison, J., et al. 2009, LSST Science Book v.2.0, https://www.1sst.org/scientists/scibook

Lupton, R., Gunn, J. E., Ivezić, Z., Knapp, G. R., \& Kent, S. 2001, in ASP Conf. Ser. 238, Astronomical Data Analysis Software and Systems X, ed. F. R. Harnden, Jr., F. A. Primini, \& H. E. Payne (San Francisco, CA: ASP), 269 Magnier, E. A., Schlafly, E., Finkbeiner, D., et al. 2013, ApJS, 205, 20

Malhotra, R. 1993, Natur, 365, 819

Malhotra, R. 1995, AJ, 110, 420

Miyazaki, S., Komiyama, Y., Kawanomoto, S., et al. 2018, PASJ, 70, S1 Murray-Clay, R. A., \& Chiang, E. I. 2005, ApJ, 619, 623

Nihei, T. C., Lehner, M. J., Bianco, F. B., et al. 2007, AJ, 134, 1596

Peixinho, N., Doressoundiram, A., Delsanti, A., et al. 2003, A\&A, 410, L29

Petit, J.-M., Kavelaars, J. J., Gladman, B. J., et al. 2011, AJ, 142, 131

Pike, R. E., Fraser, W. C., Schwamb, M. E., et al. 2017, AJ, 154, 101

Romanishin, W., \& Tegler, S. C. 1999, Natur, 398, 129

Roques, F., Moncuquet, M., \& Sicardy, B. 1987, AJ, 93, 1549

Roques, F., Moncuquet, M., Lavillonière, N., et al. 2003, ApJL, 594, L63

Ryan, E. L., Sharkey, B. N. L., \& Woodward, C. E. 2017, AJ, 153, 116

Schlafly, E. F., Finkbeiner, D. P., Jurić, M., et al. 2012, ApJ, 756, 158

Schlichting, H. E., Ofek, E. O., Sari, R., et al. 2012, ApJ, 761, 150

Shankman, C., Gladman, B. J., Kaib, N., Kavelaars, J. J., \& Petit, J. M. 2013 ApJL, 764, L2

Sheppard, S. S. 2012, AJ, 144, 169

Sheppard, S. S., \& Jewitt, D. 2004, AJ, 127, 3023

Sheppard, S. S., Lacerda, P., \& Ortiz, J. L. 2008, in The Solar System Beyond Neptune, ed. M. A. Barucci et al. (Tucson, AZ: Univ. Arizona Press), 129 Spearman, C. 1904, Am. J. Psych., 15, 72

Stellingwerf, R. F. 1978, ApJ, 224, 953

Tegler, S. C., Romanishin, W., \& Consolmagno, G. J., J. S. 2016, AJ, 152, 210

Terai, T., Yoshida, F., Ohtsuki, K., et al. 2018, PASJ, 70, S40

The Astropy Collaboration, Price-Whelan, A. M., Sipőcz, B. M., et al. 2018, AJ, 156, 123

Thirouin, A., Noll, K. S., Ortiz, J. L., \& Morales, N. 2014, A\&A, 569, A3

Thirouin, A., Ortiz, J. L., Duffard, R., et al. 2010, A\&A, 522, A93

Thirouin, A., \& Sheppard, S. S. 2017, AJ, 154, 241

Thirouin, A., \& Sheppard, S. S. 2018, AJ, 155, 248

Thirouin, A., Sheppard, S. S., Noll, K. S., et al. 2016, AJ, 151, 148

Thirouin, A., Sheppard, S. S., \& Noll, K. S. 2017, ApJ, 844, 135

Thommes, E. W., Duncan, M. J., \& Levison, H. F. 2002, AJ, 123, 2862

Tody, D. 1993, in ASP Conf. Ser. 52, Astronomical Data Analysis Software and Systems II, ed. R. J. Hanisch, R. J. V. Brissenden, \& J. Barnes (San Francisco, CA: ASP), 173

Tonry, J. L., Stubbs, C. W., Lykke, K. R., et al. 2012, ApJ, 750, 99

Trujillo, C. A., \& Brown, M. E. 2002, ApJL, 566, L125

Tsiganis, K., Gomes, R., Morbidelli, A., \& Levison, H. F. 2005, Natur, 435,459

van der Walt, S., Colbert, S. C., \& Varoquaux, G. 2011, CSE, 13, 22

van Rossum, G., \& de Boer, J. 1991, Proc. Spring 1991 EurOpen Conf., EurOpen: UNIX Distributed Open Systems in Perspective (Buntingford: EurOpen), 229

Zhang, Z.-W., Lehner, M. J., Wang, J.-H., et al. 2013, AJ, 146, 14 